October 1992

SISSA $/ 180 / 92 / F M$

\title{
Selberg Supertrace Formula for Super Riemann Surfaces III: Bordered Super Riemann Surfaces
}

\author{
by \\ Christian Grosche \\ Scuola Internazionale Superiore di Studi Avanzati \\ International School for Advanced Studies \\ Via Beirut 4 \\ 34014 Trieste, Miramare, Italy
}

\begin{abstract}
This paper is the third in a sequel to develop a super-analogue of the classical Selberg trace formula, the Selberg supertrace formula. It deals with bordered super Riemann surfaces. The theory of bordered super Riemann surfaces is outlined, and the corresponding Selberg supertrace formula is developed. The analytic properties of the Selberg super zeta-functions on bordered super Riemann surfaces are discussed, and super-determinants of Dirac-Laplace operators on bordered super Riemann surfaces are calculated in terms of Selberg super zeta-functions.
\end{abstract}





\section{Introduction}

It took a long time before physicists acknowledged the true value of the Selberg trace formula as introduced by A.Selberg in his famous paper [63]. The original attempt of Selberg to formulate his trace formula was based on number theoretical considerations, and in fact, there is a close relation between the areas of analytic number theory, eigenvalues of Laplacians on Riemann surfaces, and the Selberg trace formula (see e.g. [37, 64]), and in particular Selberg was interested to study the analytic properties of a function closely related to the trace formula, the Selberg zeta-function.

Physicists, however, have other objectives: they want to learn something about the spectrum of a model, or they want to calculate determinants, say. The latter approach to the use of the Selberg trace formula appears in the quantum field theory on Riemann surfaces, i.e. in the Polyakov approach [19-22, 56, 57] to (bosonic-, fermionic- and super-) string theory. In the perturbation expansion of the Polyakov path integral one is left with a summation over all topologies of world sheets a string can sweep out, and an integral over the moduli space of Riemann surfaces. This picture is true for bosonic strings (BS) as well as for fermionic strings (FS). The partition function turns out to be for open as well as closed bosonic strings corresponding to a topology without conformal Killing vectors (Blau and Clements [10], and D'Hoker and Phong [19, 22])

$$
Z_{0}^{(B S)}=\sum_{g} \int d \mu_{W P}\left[\operatorname{det}\left(P_{1}^{\dagger} P_{1}\right)\right]^{1 / 2}\left(\operatorname{det}^{\prime} \Delta_{0}\right)^{-D / 2} .
$$

$P_{1}$ and $\Delta_{0}$ are the symmetrized traceless covariant derivative and scalar Laplacian with Dirichlet boundary-conditions, respectively, and $d \mu_{W P}$ denotes the Weil-Petersen measure. $D$ denotes the critical dimension which equals 26 for the bosonic string. For the fermionic-, respectively the super-string all quantities have to be replaced by their appropriate super case, and the critical dimension $D=10$.

The calculation of (super-) determinants of Laplacians on closed Riemann surfaces is due to several authors. Mainly two approaches must be mentioned, firstly the evaluation of these determinants in terms of Selberg zeta-functions, e.g. Baranov, Manin et al. [47], Bolte and Steiner [13], D'Hoker and Phong [20, 22], Efrat [24], Ref.[33], Gilbert [27], Namazie and Rajjev [51], Steiner [66], and Voros [73], and secondly in terms of the period matrix and theta-functions, the most important are Alvarez-Gaumé et al. [2] and Manin [45]. Formal as these results may be, the expressions in terms of Selberg zeta-functions provide tools to investigate the convergence, respectively divergence properties of the string path integral a là Polyakov, hence non-perturbative statements are possible. In the bosonic string theory, this approach enabled Gross and Periwal [36] to show that the perturbation expansion for the closed bosonic string is not Borel-summable, and hence not finite; this statement can be easily generalized to open bosonic strings [12]. In the fermionic string theory a better asymptotic behaviour is expected, however, due to the almost unknown structure of the corresponding super moduli space, the arguing of Gross and Periwal cannot be taken over in an obvious way.

In the perturbation expansion of the bosonic string the classical Selberg trace formula could be applied in a straightforward way; the perturbation theory of the fermionic string $[21,22]$ required the devolving of a super-analogue of the classical Selberg trace formula, i.e. the Selberg supertrace formula. Here Baranov, Manin et al. [4-7] originally started this business, and it was further developed by Aoki [3] and in Refs. [33, 34], and see [35] for a short review of some recent results. 
It was mainly the closed string theory that was dealt with and for which the whole perturbation theory for scattering amplitudes was developed quite comprehensively. The case of the open bosonic, respectively open fermionic string, starting with the pioneering work of Alvarez [1], took somewhat longer and seems until now not such as good developed as the former.

Contributions along the lines of the Polyakov path integral approach for the open string theory are due to e.g. Blau et al. [10, 11], Bolte and Grosche [12], Bolte and Steiner [14], Burgess and Morris [15], Carlip [16], Dunbar [23], Jaskolski [41], Luckock [44], MartínDelgado and Mittelbrunn [46], Ohndorf [53], Rodrigues et al. [17, 59, 60], and Wu [74].

Of course, while dealing with open strings one has to distinguish Dirichlet and Neumann boundary-conditions, respectively. Here again we have two possibilities to express determinants either by the period-matrix, or by appropriate chosen Selberg zeta-functions for the corresponding Dirichlet or Neumann boundary-condition problems. The former was discussed by Burgess and Morris [15], Dunbar [23], Losev [43], Luckock [44], MartínDelgado and Mittelbrunn [46], Rodrigues and Van Tander [60] (in particular to give explicit expression on the one-loop level), and Mozorov and Rosly [50] for multiloop expressions. The latter case was approached by Refs.[10-12, 14].

In particular all the cited authors could derive relations between the determinants $\operatorname{det} \Delta_{\Sigma}^{(D)}$ and $\operatorname{det}^{\prime} \Delta_{\Sigma}^{(N)}$ corresponding to Dirichlet and Neumann boundary-conditions on the bordered Riemann surface $\Sigma$ on the one hand, and the determinant of the scalar Laplacian $\operatorname{det}^{\prime} \Delta_{\widehat{\Sigma}}$ for the doubled (closed) Riemann surfaces $\widehat{\Sigma}$ on the other, i.e. (the prime denotes the omission of possible zero-modes)

$$
\operatorname{det} \Delta_{\Sigma}^{(D)} \cdot \operatorname{det}^{\prime} \Delta_{\Sigma}^{(N)}=\operatorname{det}^{\prime} \Delta_{\widehat{\Sigma}}
$$

Whereas all this deals only with the bosonic string with boundaries, the case of the incorporation of spin-structures and fermionic strings with boundaries seems quite poorly developed.

The Selberg trace formula for bordered Riemann surfaces does exist and almost the entire theory is due to Venkov [70-72], including particular cases [69]. Independently, later on the Selberg trace formula for bordered Riemann surfaces was discussed by Blau and Clements [10], Bolte and Grosche [12] and Bolte and Steiner [14].

Let us assume that the generating functional in the theory of the open fermionic string can also be expressed as $[21,22]$

$$
Z_{0}^{(F S)}=\sum_{g} \int d \mu_{s W P}\left[\operatorname{sdet}\left(P_{1}^{\dagger} P_{1}\right)\right]^{1 / 2}\left(\operatorname{sdet}^{\prime} \square_{0}^{2}\right)^{-5 / 2},
$$

where $P_{1}$ and $\square_{0}$ are the super-analogues of the symmetrized traceless covariant derivative and scalar Dirac-Laplace operator with Dirichlet boundary-conditions, respectively, and $d \mu_{s W P}$ denotes the super Weil-Petersen measure. In order to deal with the vector DiracLaplace operator $P_{1}^{\dagger} P_{1}$ the incorporation of $m$-weighted super automorphic forms into the formalism is required. 
The development of the fermionic string, respectively the super-string model [29$32,62]$ was enormously boosted by the discovery of particular anomaly-free properties of certain gauge groups by Green and Schwarz [31]. In two previous publications, hereafter denoted as I [33], and II [34], respectively, I have already discussed various aspects of the Selberg supertrace formula on super Riemann surfaces. In Ref.[33] the Selberg supertrace formula for hyperbolic conjugacy classes was developed in full detail, including an analysis of the properties of the Selberg super zeta-functions and super-determinants of DiracLaplace operators on Super Riemann surfaces in order to discuss the fermionic string integrand in the Polyakov path integral properly. In Ref.[34], I continued these studies by the incorporation of elliptic and parabolic conjugacy classes. However, to complete a comprehensive development of the Selberg supertrace formula, bordered super Riemann surfaces must be included in the discussion. Bordered super Riemann surfaces, of course, occur for open (fermionic- and super-) strings (in the case of super-strings, so called type I super strings with the $O(32)$ gauge group).

The further contents will be now as follows:

In the second section I give a short introduction into the theory of bordered Riemann surfaces and indispensible information concerning the spectral theory on bordered Riemann surfaces. Information concerning the definition of super Riemann surfaces was already given in Refs.[33, 34], but is included here to make the paper self-contained. The citation of the Selberg trace formula on bordered Riemann surfaces is also included.

The third section sets up a proposal to define bordered super Riemann surfaces. Concerning the theory of super Riemann surfaces I refer to the relevant literature, only the most important relations and indispensible ingredients will be given.

The fourth section attacks the actual derivation of the Selberg supertrace formula for bordered super Riemann surfaces. After the incorporation of the hyperbolic conjugacy classes, the usual elliptic ones cause no difficulty and the result of Ref.[34] can be taken over without delay; the incorporation of the parabolic conjugacy classes (Dirichlet boundaryconditions) requires some care and a regularization procedure is needed. The incorporation of Neumann boundary-conditions then follows by a proper combination of the former results.

In the fifth section, I discuss the analytic properties of the (modified) Selberg super zeta-functions on bordered super Riemann surfaces by means of the supertrace formula.

In the sixth section, the results of the former two are applied to the problem of calculating super-determinants of Dirac-Laplace operators on bordered super Riemann surfaces. As explicit as possible expressions are evaluated in terms of the Selberg super zeta-functions on bordered super Riemann surfaces.

All the principle results will be stated as theorems.

The last section is devoted to a summary and a discussion of the results.

\section{The Selberg Trace Formula on Bordered Riemann Surfaces}

1. Bordered Riemann surfaces and the construction of the Selberg operator. We start with usual bordered Riemann surfaces (we rely on Sibner [65] and Venkov [71], compare also Refs.[12, 14]). Let us consider a Riemann $\widetilde{\Sigma}$ surface of genus $g$ and $d_{1}, \ldots, d_{n}$ conformal, non-overlapping discs on $\widetilde{\Sigma}$. Then $\Sigma=\widetilde{\Sigma} \backslash\left\{d_{1}, \ldots, d_{n}\right\}$ is a bordered Riemann surface with Dirichlet, respectively Neumann boundary-conditions of signature $(g, n) . c_{i}=\partial d_{i}$ are the $n$ components of $\partial \Sigma$. Now take a copy $\mathcal{I} \Sigma$ of $\Sigma$, a mirror image, and glue both together along $\partial \Sigma$ and $\partial \mathcal{I} \Sigma$. Explicitly this can be realized by taking the refection $\mathcal{I}$ to have the form $\mathcal{I} z \rightarrow z^{\prime}=-\bar{z}(z \in \mathcal{H})$. The reflection $\mathcal{I}$ in $\partial \Sigma$ then is a anti-conformal 
involution $\left(\mathcal{I}^{2}=1\right)$ on the doubled surface $\widehat{\Sigma}=\Sigma \cup \mathcal{I} \Sigma . \widehat{\Sigma}$ is a Riemann surface of genus $\widehat{g}=2 g+n-1$, and we set $\widehat{\mathcal{A}}=\mathcal{A}(\widehat{\mathcal{F}})$. The uniformization theorem for Riemann surfaces now yields that $\widehat{\Sigma}$ may be represented as $\widehat{\Sigma} \cong \widehat{\Gamma} \backslash \mathcal{H}$, where $\widehat{\Gamma}$ is the Fuchsian group of $\widehat{\Sigma}$. Of course there is a fundamental domain $\widehat{\mathcal{F}}$ of $\widehat{\Sigma}$ in $\mathcal{H}$, tesselating $\mathcal{H}$. In order to construct a convenient fundamental domain and representation of the involution $\mathcal{I}$, we view according to Sibner [65] and Venkov [71] $\widehat{\Sigma}$ as a symmetric Riemann surface with reflection symmetry $\mathcal{I}$. Then $\widehat{\mathcal{F}}$ may be chosen as the interior of a fundamental polygon with $4 \widehat{g}+2 n-2$ edges which is symmetric with respect to the imaginary axis. Due to the explicit choice of $\mathcal{I}$ as $\mathcal{I} z=-\bar{z}$, one of the bordering curves, say $c_{1}$, is mapped onto the imaginary axis and the others among the edges of the fundamental polygon. With this construction one can work directly on $\widehat{\mathcal{F}}$, with $\mathcal{I}$ viewed as a mapping of complex numbers, being formally identical on $\widehat{\Sigma}$ and $\widehat{\mathcal{F}}$.

In the case that elliptic fixed points and cusps are present, the non-euclidean area of a Riemann surface is given by (e.g. [39], Gauss-Bonnet theorem)

$$
\mathcal{A}=2 \pi\left[2(g-1)+\kappa+\sum_{j=1}^{s}\left(1-\frac{1}{\nu_{j}}\right)\right],
$$

where $s$ denotes the number of inequivalent elliptic fixed points and $\kappa$ the number of inequivalent cusps (i.e. the number of zero interior angles of the fundamental polygon $\mathcal{F}$ ). $\nu_{j}$ denotes the order of the elliptic generators $R_{j} \subset \Gamma(1 \leq j \leq s)$, i.e. $R_{j}^{\nu_{j}}=1$ for $\left(1 \leq j \leq s, 1<\nu_{j}<\infty\right)$.

In order to set up our notation we start by citing some results of the classical Selberg trace formula for bordered Riemann surfaces [71]. As usual one starts by formulating the appropriate automorphic kernel. Consequently this gives for automorphic functions the property $f(\gamma z)=\chi_{\gamma}^{m} j(\gamma, z) f(z)(\gamma \in \widehat{\Gamma})$, the inversion $f(\mathcal{I} z)= \pm f(z)$ which distinguishes even and odd automorphic functions with respect to $x . \quad \chi(\gamma) \equiv \chi_{\gamma}$ denotes a multiplier system acting according to $\bar{\Gamma} \rightarrow\{ \pm 1\}$ with $\chi(-\mathbb{1})=-1(\gamma \in \bar{\Gamma}$, such that $\bar{\Gamma} \in \operatorname{SL}(2, \mathbb{R})$, $\widehat{\Gamma}=\bar{\Gamma} /\{ \pm 1\})$, and $j(\gamma, z)$ denotes the automorphic weight $[12,38]$.

The automorphic kernel is then constructed as follows $[12,14,71]$

$$
\widehat{K}_{N, D}(z, w)=\frac{1}{2} \sum_{\{\gamma\}}[k(z, \gamma w) \pm k(z, \gamma \mathcal{I} w)],
$$

where the "+"-sign stands for Neumann, and the "-"-sign for Dirichlet boundary-conditions. $\sum_{\{\gamma\}}$ denotes the summation over distinct conjugacy classes. Let $L$ the Selbergoperator with eigenvalue $\Lambda(\lambda)$ on $\mathcal{F}$ (where $k(z, w)$ is the corresponding integral kernel) together with its counterpart $\widehat{L}$ on $\widehat{\mathcal{F}}$, and we introduce the Maass-Laplacian $D_{m}=$ $-y^{2}\left(\partial_{x}^{2}+\partial_{y}^{2}\right)+i m y \partial_{x}$ on $\mathcal{F}$ in $\mathcal{L}^{2}(\mathcal{F}, m, \chi) \equiv \mathcal{L}^{2}(\mathcal{F})$ (the space of square integrable automorphic forms).

To each cusp there is associated an Eisenstein series

$$
e(z, s, \alpha)=\sum_{\gamma \in \Gamma_{\alpha} \backslash \Gamma} y^{s}(\gamma z)
$$

$z \in \mathcal{H}, \Re(s)>1, \alpha=1, \ldots, \kappa$, with $\Gamma_{\alpha}$ the stabilizer of the cusp $\alpha$. In the spectral decomposition of $D_{m}$ on $\mathcal{L}^{2}(\mathcal{F})$ these Eisenstein series span the continuous spectrum. 
Let us start with Dirichlet boundary-conditions and only the odd automorphic functions with respect to $x$ survive in the spectral expansion of the automorphic kernel. Let $f \in$ $\mathcal{L}^{2}(\mathcal{F})$ an odd eigenfunction in $x$ of $D_{m}$ with eigenvalue $\lambda$. A glance on the continuous spectrum shows that the Eisenstein series $e(z, s)$ drop out, c.f. according to a theorem by Venkov [71]. Then

$$
\begin{aligned}
\left(\widehat{L}_{D} f\right)(z) & =\frac{1}{2} \int_{\widehat{\mathcal{F}}} \widehat{K}_{D}\left(z, z^{\prime}\right) f\left(z^{\prime}\right) d V\left(z^{\prime}\right) \\
& =\frac{1}{2}(L f)(z)-\frac{1}{2}(L f)(-\bar{z})=\Lambda(\lambda) f(z)
\end{aligned}
$$

if $f \in \mathcal{L}^{2}(\mathcal{F})$ even, similarly for Neumann boundary-conditions

$$
\begin{aligned}
\left(\widehat{L}_{N} f\right)(z) & =\frac{1}{2} \int_{\widehat{\mathcal{F}}} \widehat{K}_{N}\left(z, z^{\prime}\right) f\left(z^{\prime}\right) d V\left(z^{\prime}\right) \\
& =\frac{1}{2}(L f)(z)+\frac{1}{2}(L f)(-\bar{z})=\Lambda(\lambda) f(z) .
\end{aligned}
$$

In the case of Dirichlet boundary-conditions we are thus left with the spectral expansion of the automorphic kernel into odd discrete eigenfunctions $\Psi_{n}$ on $\mathcal{H}$

$$
\widehat{K}_{D}(z, w)=\sum_{n} h\left(p_{n}\right) \Psi_{n}(z) \Psi_{n}(w)
$$

where $\Lambda\left(\lambda_{n}\right)=\Lambda\left(p_{n}^{2}+\frac{1}{4}\right) \equiv h\left(p_{n}\right)$. In the case of Neumann boundary-conditions we get the spectral expansion of the automorphic kernel into even discrete and continuous eigenfunctions $\Phi_{n}$ and $e(z, s)$, respectively, on $\mathcal{H}$

$$
\widehat{K}_{N}(z, w)=\sum_{n} h\left(p_{n}\right) \Phi_{n}(z) \Phi_{n}(w)+\frac{1}{4 \pi} \int_{-\infty}^{\infty} h(p) e\left(z, \frac{1}{2}+i p\right) \overline{e\left(w, \frac{1}{2}+i p\right)}
$$

2. Conjugacy classes on bordered Riemann surfaces and the trace formula. As usual the quantity $N_{\gamma}$ is called norm of an hyperbolic $\gamma \in \Gamma$ and $N_{\gamma_{0}}$ will denote the norm of a primitive hyperbolic $\gamma \in \Gamma$, and $l_{\gamma}=\ln N_{\gamma}$ denotes the length corresponding to a $\gamma \in \Gamma$. $\gamma_{0} \in \Gamma$ is called a primitive element, if it is not a power of any other element of $\Gamma$. Each element $\gamma \in \Gamma /\{ \pm \mathbb{1}\}$ is thus uniquely described as $\gamma=k^{-1} \gamma_{0} k$ for some primitive $\gamma_{0}, n \in \mathbb{N}$ and $k \in \Gamma / \Gamma_{\gamma_{0}}$. Conjugacy classes are defined by $\{\gamma\}:=\left\{\gamma_{i} \in \Gamma \mid \gamma_{i}=k^{-1} \gamma k, k \in \Gamma\right\} . \sum_{\{\gamma\}}$ denotes the summation over primitive conjugacy classes of a particular conjugacy class $\{\gamma\}$ within the Fuchsian group $\widehat{\Gamma}$. In the following I will omit the index " 0 " in $\gamma_{0}$ if it is obvious that indeed the primitive $\gamma_{0}$ is meant and no confusion can arise.

Let $\kappa$ be the number of inequivalent cups on the fundamental domain $\widehat{\mathcal{F}}$ (the number of primitive parabolic conjugacy classes in $\widehat{\Gamma}$ ), and by $\Gamma_{\rho}$ the restriction of $\widehat{\Gamma}$ to this inversion counterpart, where I have abbreviated $\rho:=\gamma \mathcal{I}$. In order to investigate the various conjugacy classes for the formulation of the Selberg trace-formula for bordered Riemann surfaces, we have to distinguish the original conjugacy classes which appear already for closed Riemann surfaces and additional conjugacy classes due to $\gamma \mathcal{I}$. The new 
conjugacy classes can be characterized by their traces. We consider first compact Riemann surfaces, i.e. compact polygons as fundamental domains. The case of the closed Riemann surfaces gives us hyperbolic and elliptic conjugacy classes which correspond to $|\operatorname{tr}(\gamma)|>2$, respectively $|\operatorname{tr}(\gamma)|<2$.

In the theory of symmetric spaces it is convenient to consider the following isomorphic model of $\mathcal{H}$. One defines the positive definite symmetric matrices

$$
z(x ; y)=\left(\begin{array}{cc}
y+x / y^{2} & x / y \\
x / y & 1 / y
\end{array}\right), \quad(x \in \mathbb{R}, y>0) .
$$

If $g \in \mathrm{SL}(2, \mathbb{R})$, then the group action has the form

$$
g z(x ; y)=g[z(x ; y)] g^{t},
$$

where $g^{t}$ denotes the transpose of $g$. In this model it is easy to implement the involution $\mathcal{I}$ in terms of the matrix

$$
\mathcal{I}=\left(\begin{array}{cc}
1 & 0 \\
0 & -1
\end{array}\right)
$$

i.e. $\mathcal{I}$ is an element in $\operatorname{GL}(2, \mathbb{R}) /\{ \pm \mathbb{1}\}$.

Within this model we find, first, for $\operatorname{tr}(\rho) \neq 0$ that the relative centralizer is of the form

$$
\left(\begin{array}{cc}
b & 0 \\
0 & -b^{-1}
\end{array}\right), \quad(\bmod \pm 1)
$$

[Centralizers $\Gamma_{\gamma}$ are defined by $\left.\Gamma_{\gamma}:=\left\{\gamma_{i} \in \Gamma \mid \gamma_{i}^{-1} \gamma \gamma_{i}=\gamma\right\}\right]$. Therefore $\Gamma \mathcal{I} \subset \widehat{\Gamma}$ consists of hyperbolic elements and the identity and since $\widehat{\Gamma}$ is discrete of a single hyperbolic element. The second case gives $\operatorname{tr}(\rho)=0$. Then the relative centralizer consists of elements of the form

$$
\rho_{1}=\left(\begin{array}{cc}
c & 0 \\
0 & c^{-1}
\end{array}\right), \quad \rho_{2}=\left(\begin{array}{cc}
0 & d \\
-d^{-1} & 0
\end{array}\right), \quad(\bmod \pm 1) .
$$

$\rho_{2}$ is an elliptic element of order two. Thus $\gamma \mathcal{I}$ consists of hyperbolic, elliptic and the identity element. However, due to the construction $\rho_{1}^{n} \rho_{2}(n \in \mathbb{Z})$ we see that we can generate infinitely many elliptic conjugacy classes which is impossible, since $\widehat{\Gamma}$ is discrete. Therefore the relative centralizer of $\gamma \mathcal{I}$ with $\operatorname{tr}(\gamma \mathcal{I})=0$ consists either of hyperbolic elements and the identity or by a single elliptic generator of order two. The explicit computation reveals that in the compact case only the former case is possible, the latter leading to a divergency.

The conjugacy classes of $\rho \in \widehat{\Gamma} \mathcal{I}$ can therefore be distinguished in two ways $[10,12$, 14] according to their squares $\rho^{2} \in \widehat{\Gamma}$. Let $\rho \in \widehat{\Gamma}$ be primitive, that is not a positive power of any other element of $\widehat{\Gamma} \mathcal{I}$. Then

i) $\rho=\rho_{i}, \rho_{i}^{2} \in\left\{C_{i}\right\}_{\widehat{\Gamma}}, i=1, \ldots, n$. The $\left\{C_{i}\right\}_{\widehat{\Gamma}}$ are the conjugacy classes of the $C_{i}$ in $\widehat{\Gamma}$ which correspond to the closed geodesics $c_{i}$ on $\widehat{\Sigma}$.

ii) $\rho=\rho_{p}, \rho_{p}^{2}$ being a primitive element in $\widehat{\Gamma}$ and $\rho_{p}^{2} \neq\left\{C_{i}\right\}_{\widehat{\Gamma}}$.

In the notation of Venkov [71] the relative hyperbolic conjugacy classes with $\{\rho\}$ with $\operatorname{tr}(\rho)=0$ correspond to the case i), and the relative hyperbolic conjugacy classes with $\{\rho\}$ with $\operatorname{tr}(\rho) \neq 0$ correspond to the case ii). 
Thus it follows that the sum over conjugacy classes for $\rho \in \widehat{\Gamma} \mathcal{I}$ is divided into first the conjugacy classes of the $C_{i}$ in $\widehat{\Gamma}$, which correspond to the closed geodesics $c_{i}$ on $\widetilde{\Sigma}$, and second into conjugacy classes such that for all $\rho \in \widehat{\Gamma} \mathcal{I}$ there is a unique description $\gamma=$ $k^{-1} \rho^{2 n-1} k(n \in \mathbb{N})$, for $\rho \in \widehat{\Gamma} \mathcal{I}$ inconjugate and primitive, and $k \in \Gamma_{\rho^{2}} \backslash \widehat{\Gamma}$.

Let us continue by considering a non-compact polygon. The additional conjugacy classes are again classified according to their trace. The conjugacy class $\operatorname{tr}(\gamma)=2$ with corresponding non-compact Fuchsian group $\widehat{\Gamma}$ gives the already known parabolic conjugacy classes. Each conjugacy classes with $\operatorname{tr}(\rho)=0$ give rise to an elliptic transformation whose centralizer is generated by a single generator of order two (see above). In other words, for each $\gamma \in \widehat{\Gamma}$ there exists an element $g \in \operatorname{PSL}(2, \mathbb{R})$ having the properties

$$
g \rho g^{-1}=\mathcal{I}, \quad g \widehat{\Gamma}_{\rho} g^{-1}\left\{\mathbb{1}_{2},\left(\begin{array}{cc}
0 & a \\
-1 / a & 0
\end{array}\right)(\bmod \pm 1)\right\},
$$

where $a \geq 1$. These classes play the rôle of the parabolic classes in the classical Selberg trace formula. Evaluating all contributions, we can write down

Theorem 2.1 [12, 14, 71]: The Selberg trace formula on arbitrary bordered Riemann surfaces for automorphic forms of weight $m, m \in \mathbb{Z}$, is given by

$$
\begin{aligned}
& \sum_{n=1}^{\infty} h\left(p_{n}\right)=-\frac{\widehat{\mathcal{A}}}{16 \pi^{2}} \int_{0}^{\infty} \frac{\cosh \frac{u m}{2}}{\sinh \frac{u}{2}} g^{\prime}(u) d u+\frac{1}{4} \sum_{\{\gamma\}} \sum_{k=1}^{\infty} \frac{\chi_{\gamma}^{m k} l_{\gamma} g\left(k l_{\gamma}\right)}{\sinh \frac{k l_{\gamma}}{2}} \\
& +\frac{i}{4} \sum_{\{R\}} \sum_{k=1}^{\nu-1} \chi_{R}^{m k} \frac{e^{i(m-1) k \pi / \nu}}{\nu \sin (k \pi / \nu)} \int_{-\infty}^{\infty} d u g(u) \frac{e^{(m-1) u / 2}\left(e^{u}-e^{2 i k \pi / \nu}\right)}{\cosh u+\cos [\pi-2(k \pi / \nu)]} \\
& -\frac{1}{4} \sum_{\left\{\rho^{2}\right\}} \sum_{k=0}^{\infty} \frac{\chi_{\rho}^{m(2 k+1)} \chi_{\mathcal{I}}^{m} l_{\rho^{2}} g\left[\left(k+\frac{1}{2}\right) l_{\rho^{2}}\right]}{\cosh \left[\frac{1}{2}\left(k+\frac{1}{2}\right) l_{\rho^{2}}\right]}-\frac{1}{2} \sum_{i=1}^{n} \sum_{k=1}^{\infty} \frac{\chi_{C_{i}}^{m k} l_{C_{i}} g\left(k l_{C_{i}}\right)}{\cosh \frac{k l_{C_{i}}}{2}} \\
& +\frac{g(0)}{2}\left[\frac{1}{4} \sum_{\substack{\{\rho\} ; \widehat{\Gamma}_{\rho, e l l} \\
\operatorname{tr}(\rho)=0}} \chi_{\rho}^{m} \ln \left(\frac{a(\rho)}{\nu(\rho)}\right)-\kappa_{0} \ln 2-\frac{L}{2}\right]+\frac{\kappa_{0}}{8} h(0) \\
& -\frac{\kappa_{0}}{4 \pi} \int_{-\infty}^{\infty} h(p) \Psi\left(\frac{1}{2}+i p\right) d p+\frac{\kappa_{0}}{4} \int_{0}^{\infty} \frac{g(u)}{\sinh \frac{u}{2}}\left(1-\cosh \frac{u m}{2}\right) d u .
\end{aligned}
$$

with the abbreviation $L=\sum_{i=1}^{n} l_{C_{i}}$ and where the $\lambda_{n}=\frac{1}{4}+p_{n}^{2}$ on the left run through the set of all eigenvalues of the Dirichlet problem, and the summation on the right is taken over all primitive conjugacy classes $R \in \widehat{\Gamma}$ with $\operatorname{tr}(R)<2, \gamma \in \widehat{\Gamma}$ with $\operatorname{tr}(\gamma)>2$, and $\gamma \mathcal{I} \in \widehat{\Gamma}, \operatorname{tr}(\rho) \neq 0$. The lengths $l_{C_{i}}$ are twofold degenerate, since $C_{i}$ and $C_{i}^{-1}$ both have to be included into the sum.

$h(p)$ denotes an even function in $p$ and must has the following properties

i) $h(p)$ is holomorphic in the strip $|\Im(p)| \leq \frac{1}{2}+\epsilon, \epsilon>0$.

ii) $h(p)$ has to decrease faster than $|p|^{-2}$ for $p \rightarrow \pm \infty$.

iii) $g(u)=\pi^{-1} \int_{0}^{\infty} h(p) \cos (\pi p) d p$. 
Note that for Neumann boundary-conditions the inverse-hyperbolic terms change their signs. In this case, however, the parabolic terms are quite different, due to the additional presence of the continuous spectrum represented by Eisenstein-series, see e.g. Ref.[39]. $a(\rho)$ and $\mu(\rho)$ are quantities specific to the conjugacy class of the elliptic $\gamma \in \widehat{\Gamma}$ with $\operatorname{tr}(\rho)=0$ which will be explained later on [c.f. Theorem 4.2], and we require the following property of the multiplier system

$$
\kappa_{0}:=\sum_{\{S\}} \chi_{S}^{m}=\sum_{\substack{\{\rho\} ; \widehat{\Gamma}_{\rho, e l l} \\ \operatorname{tr}(\rho)=0}} \chi_{\rho}^{m} .
$$

\section{Super Riemann Surfaces, Bordered Super Riemann Surfaces and the Selberg Supertrace Formula on Closed Super Riemann Surfaces}

1. Super Riemann surfaces and construction of bordered super Riemann surfaces. We sketch some important facts about super Riemann surfaces. For more details I refer to Batchelor et al. [8, 9], DeWitt [18], Moore, Nelson and Polchinski [49], Ninnemann [52], Rabin and Crane [58], and Rogers [61]. Let us start with a (1|1) (complex)-dimensional (not necessarily) flat superspace, parameterized by even coordinates $Z \in \mathbb{C}_{c}$ and odd (Grassmann) coordinates $\theta \in \mathbb{C}_{a}$, respectively. Let $\Lambda_{\infty}$ be the infinite dimensional vector space generated by elements $\zeta_{a}(a=1,2, \ldots)$ with basis $1, \zeta_{a}, \zeta_{a} \zeta_{b}, \ldots(a<b)$ and the anticommuting relation $\zeta_{a} \zeta_{b}=-\zeta_{b} \zeta_{a}, \forall_{a, b}$. Every $Z \in \Lambda_{\infty}$ can be decomposed as $Z=$ $Z_{B}+Z_{S}$ with $Z_{B} \in \mathbb{C}_{c} \equiv \mathbb{C}, Z_{S}=\sum_{n} \frac{1}{n !} c_{a_{1}, \ldots, a_{n}} \zeta^{a_{n}} \ldots \zeta^{a_{1}}$, with the $c_{a_{1}, \ldots, a_{n}} \in \mathbb{C}_{a}$ totally antisymmetric. $Z_{B}$ and $Z_{S}$, respectively, are called the body (sometimes denoted by $Z_{B}=Z_{\text {red }}$ ) and soul of the supernumber $Z$, respectively. The notion of superspace and supermanifolds as introduced by Batchelor and Bryant [8, 9], DeWitt [18], Rabin and Crane [58], and Rogers [61] enables one to represent supersymmetry transformations as pure geometric transformations in the coordinates $Z=(z, \theta) \in \mathbb{C}_{c} \times \mathbb{C}_{a}$. As is well-known, a usual complex manifold of complex dimension equal to one is already a Riemann surface. The definition of a super Riemann surface, however, requires the introduction of a superconformal structure. Let us consider the operator $D=\theta \partial_{z}+\partial_{\theta}$ (note $D^{2}=\partial_{z}$ ). Further we consider a general superanalytic coordinate transformation $\widetilde{z}=\widetilde{z}(z, \theta), \widetilde{\theta}=\widetilde{\theta}(z, \theta)$. A superanalytic coordinate transformation is called superconformal, iff the (0|1)-dimensional subspace of the tangential space generated by the action of $D$ is invariant under such a coordinate transformation, i.e. $D=(D \widetilde{\theta}) \widetilde{D}$. This means that a coordinate transformation is super-conformal iff $D z^{\prime}=\theta^{\prime} D \theta^{\prime}$.

To study supersymmetric field theories one needs even and odd superfields. Here now the definition of DeWitt [18] of super Riemann manifolds conveniently comes into play. The infinity dimensional algebra $\Lambda_{\infty}$ supplies all the required quantities. Domains in $\mathbb{C}^{(1 \mid 1)}$ with coordinates $(z, \theta)$ are constructed in such a way that the entire Grassmann algebra are attached to the usual complex coordinates. If one considers the universal family of DeWitt super Riemann manifolds with genus $g$, then only $2 g-2$ parameters of $\Lambda_{\infty}$ are required, the remaining ones are redundant.

An important property we need in our investigations is, when a supermanifold is split. This means that for a coordinate transformation $Z \rightarrow Z^{\prime}\left(Z, Z^{\prime} \in \Lambda_{\infty}\right)$ the coefficient 
functions do not mix which each other. Let $x$ be usual local coordinates, and $\zeta \in \Lambda_{\infty}$ local Grassmann coordinates, then if a supermanifold is split then there is a global isomorphism such that the coefficient functions $y$ and $\eta$ of a super-functions $F(x, \zeta)$ transform according to

$$
\left.\begin{array}{l}
y=a_{0}(x)+a_{i j}(x) \zeta^{i} \zeta^{j}+\ldots, \\
\eta=b_{1, i}(x) \zeta^{i}+b_{3, i j k}(x) \zeta^{i} \zeta^{j} \zeta^{k}+\ldots,
\end{array} \quad \rightarrow \quad \begin{array}{l}
a_{0}^{\prime}\left(x^{\prime}\right)+a_{i j}^{\prime}\left(x^{\prime}\right) \zeta^{\prime i} \zeta^{\prime j}+\ldots, \\
b_{1, i}^{\prime}\left(x^{\prime}\right) \zeta^{\prime i}+b_{3, i j k}^{\prime}\left(x^{\prime}\right) \zeta^{\prime i} \zeta^{\prime j} \zeta^{\prime k}+\ldots,
\end{array}\right\}
$$

for $Z \rightarrow Z^{\prime}$. Due to a theorem of Batchelor [8] every differentiable supermanifold is split, and in particular every complex supermanifold of dimension $(d \mid 1)$. The super Riemann surfaces in question can be seen as complex a (1|1)-dimensional supermanifold, respectively a real (2|2)-dimensional manifold, where the coordinate transformations are super-conformal mappings [58].

To generalize the uniformization theorem for Riemann surfaces to super Riemann surfaces $\mathcal{M}$, one shows that unique generalizations $\widehat{\mathbb{C}}^{(1 \mid 1)}, \mathbb{C}^{(1 \mid 1)}$ and $\mathcal{H}^{(1 \mid 1)}:=\{(z, \theta) \in$ $\left.\mathbb{C}^{(1 \mid 1)} \mid \Im(z)>0\right\}$ of simple connected Riemann surfaces exist, and endows $U=\widehat{\mathbb{C}}^{(1 \mid 1)}, \mathbb{C}^{(1 \mid 1)}$ and $\mathcal{H}^{(1 \mid 1)}$, respectively, with a super-conformal structure, such that the local coordinate transformations are super-conformal mappings [58].

In the case of non-euclidean harmonic analysis in the context of super Riemann surfaces we consider the group $\operatorname{OSp}(2, \mathbb{C})$ of super conformal automorphisms on super Riemann surfaces as a natural generalization of Möbius transformations. They have the form

$$
\begin{aligned}
\operatorname{OSp}\left(2,1 ; \mathbb{C}_{c}^{2} \times \mathbb{C}_{a}\right):=\{\gamma= & \left(\begin{array}{rrr}
a & b & \chi_{\gamma}(b \alpha-a \beta) \\
c & d & \chi_{\gamma}(d \alpha-c \beta) \\
\alpha & \beta & \chi_{\gamma}(1-\alpha \beta)
\end{array}\right) \mid a, b, c, d \in \mathbb{C}_{c} \\
& \left.\alpha, \beta \in \mathbb{C}_{a} ; a d-b c=1+\alpha \beta ; \operatorname{sdet} \gamma=\chi_{\gamma} \in\{ \pm 1\}\right\}
\end{aligned}
$$

$(\alpha, \beta$ real, with the complex conjugate rules $\overline{f+g}=\bar{f}+\bar{g}$, and $\overline{f \cdot g}=\bar{f} \cdot \bar{g})$. Its generators are the operators $L_{0}, L_{1}, L_{-1}, G_{1 / 2}$ and $G_{-1 / 2}$ of the Neveu-Schwarz sector of the super Virasoro algebra of the fermionic string. Elements $\gamma \in \operatorname{OSp}\left(2,1 ; \mathbb{C}_{c}^{2} \times \mathbb{C}_{a}\right)$ act on elements $x=\left(z_{1}, z_{2}, \xi\right) \in \mathbb{C}_{c}^{2} \times \mathbb{C}_{a} \backslash\{0\}$ by matrix multiplication, i.e. $x^{\prime}=\gamma x$. By means of a local coordinate system $(z, \theta)=\left(z_{1} / z_{2}, \xi / z_{2}\right)$ and the requirements of superconformal transformation the local coordinate transformations are fixed and the super Möbius transformations explicitly have the form $[5,33,52,58,68]$

$$
z^{\prime}=\frac{a z+b}{c z+d}+\theta \frac{\alpha z+\beta}{(c z+d)^{2}}, \quad \theta^{\prime}=\frac{\alpha+\beta z}{c z+d}+\frac{\chi_{\gamma} \theta}{c z+d} .
$$

The $\chi_{\gamma}$ with $\chi_{\gamma}= \pm 1$ lead to the description of spin structures on a super Riemann surface. The transformation factor of the $D$ operator yields to

$$
F_{\gamma}:=\left(D \theta^{\prime}\right)^{-1}=\chi_{\gamma}(c z+d+\delta \theta),
$$


with $\delta=\chi_{\gamma} \sqrt{1+\alpha \beta}(\alpha d+\beta c)$. This general super-Möbius transformation does mix the coefficient functions of superfunctions $F \in \Lambda_{\infty}$. Since we required that the super Riemann surfaces in question is split, the odd quantities $\alpha, \beta$ are not necessary and can be omitted. It is sufficient to consider transformations $\gamma \in \operatorname{OSp}(2,1)$ with $\alpha=\beta=0$ and the characters $\chi_{\gamma}$ which describe spin structures. Furthermore $\gamma$ and $-\gamma$ describe the same transformation. We thus have that the automorphisms on $\mathcal{H}^{(1 \mid 1)}$ are given by

$$
\text { Aut } \mathcal{H}^{(1 \mid 1)}=\frac{\operatorname{OSp}(2 \mid 1, \mathbb{R})}{\{ \pm \mathbb{1}\}}
$$

and a super Fuchsian group $\Gamma$ denotes a discrete subgroup of Aut $\mathcal{H}^{(1 \mid 1)}$. Therefore we obtain for the transformations $z \rightarrow z^{\prime}$ and $\theta \rightarrow \theta^{\prime}[5,52]$

$$
z^{\prime}=\frac{a z+b}{c z+d}, \quad \theta^{\prime}=\frac{\chi_{\gamma} \theta}{c z+d}
$$

[here $\left.F_{\gamma}=\chi_{\gamma}(c z+d)\right] . \quad M_{\xi=0}$ corresponds to the usual Riemann surface $M_{\text {red }}$ with some spin-structure, since a $\gamma \in \operatorname{Aut} \mathcal{H}^{(1 \mid 1)}$ is fixed by a $\operatorname{PSL}(2, \mathbb{R})$ transformation and a character $\chi_{\gamma}= \pm 1$. The properties of the odd coordinates is determined by the properties of $M_{\text {red }}$ and $\theta$ is the cut of a spinor-bundle.

2. Dirac-Laplace operators and conjugacy classes on super Riemann surfaces. We need some further ingredients. Let us introduce the quantities $N_{\gamma}$ and $l_{\gamma}$

$$
2 \cosh \frac{l_{\gamma}}{2}=N_{\gamma}^{1 / 2}+N_{\gamma}^{-1 / 2}=a+d+\chi_{\gamma} \alpha \beta
$$

$N_{\gamma}$ is called norm of an hyperbolic $\gamma \in \Gamma$ in a (general) super Fuchsian group, and $N_{\gamma_{0}}$ will denote the norm of a primitive hyperbolic $\gamma \in \Gamma$, and $l_{\gamma}=\ln N_{\gamma}$ denotes the length corresponding to a $\gamma \in \Gamma$ and all notions from the bosonic case are interpreted in a straightforward way into their super generalization. Each element $\gamma \in \Gamma /\{ \pm \mathbb{1}\}$ is thus uniquely described as $\gamma=k^{-1} \gamma_{0} k$ for some primitive $\gamma_{0}, n \in \mathbb{N}$ and $k \in \Gamma / \Gamma_{\gamma_{0}}$. For $\operatorname{OSp}(2, \mathbb{R}) /\{ \pm \mathbb{1}\}$ in homogeneous coordinates a hyperbolic transformation is always conjugate to the transformation $z^{\prime}=N_{\gamma} z, \theta^{\prime}=\chi_{\gamma} \sqrt{N_{\gamma}} \theta$, or in matrix representation

$$
\text { hyperbolic } \gamma \in \Gamma \text { conjugate to } \quad\left(\begin{array}{ccc}
N_{\gamma}^{1 / 2} & 0 & 0 \\
0 & N_{\gamma}^{-1 / 2} & 0 \\
0 & 0 & \chi_{\gamma}
\end{array}\right) \text {. }
$$

Hyperbolic transformations are also called dilatations.

The generators of a particular super Fuchsian group of a super Riemann surface with genus $g$ obey the constraint

$$
\left(\gamma_{0} \gamma_{1}^{-1} \ldots \gamma_{2 g-2} \gamma_{2 g-1}^{-1}\right)\left(\gamma_{0}^{-1} \gamma_{1} \ldots \gamma_{2 g-2}^{-1} \gamma_{2 g-1}\right)=\mathbb{1}_{2 \mid 1}
$$


In order to construct explicitly a metric on $\mathcal{H}^{(1 \mid 1)}$ one starts with the super Vierbeins in flat superspace and performs a super Weyl transformation [40] to obtain the metric $d s^{2}=d q^{a}{ }_{a} g_{b} d q^{b}$ in $\mathcal{H}^{(1 \mid 1)}$ [68]. The scalar product has the form

$$
\left(\Phi_{1}, \Phi_{2}\right)=\int_{\mathcal{H}^{(1 \mid 1)}} \frac{d z d \bar{z} d \theta d \bar{\theta}}{2 Y} \Phi_{1}(Z) \bar{\Phi}_{2}(Z) \equiv \int_{\mathcal{H}^{(1 \mid 1)}} d V(Z) \Phi_{1}(Z) \bar{\Phi}_{2}(Z),
$$

for super functions $\Phi_{1}, \Phi_{2} \in L^{2}\left(\mathcal{H}^{(1 \mid 1)}\right)$ and $Y=y+i \theta \bar{\theta} / 2=y+\theta_{1} \theta_{2}\left(\theta=\theta_{1}+i \theta_{2}\right)$. We have one even and one odd point pair invariant given by $[5,48,68]$

$$
\begin{aligned}
R(Z, W)= & \frac{|z-w-\theta \nu|^{2}}{Y V} \\
r(Z, W)= & i \frac{2 \theta \bar{\theta}+(\nu+\bar{\nu})(\theta-\bar{\theta})}{4 Y}+i \frac{2 \nu \bar{\nu}+(\theta+\bar{\theta})(\nu-\bar{\nu})}{4 V} \\
& +\frac{(\nu-\bar{\nu})(\theta-\bar{\theta}) \Re(z-w-\theta \nu)}{4 Y V} \\
= & \frac{\left(\theta_{1}-\nu_{1}\right) \theta_{2}}{y}+\frac{\left(\nu_{1}-\theta_{1}\right) \nu_{2}}{v}+\frac{\theta_{2} \nu_{2} \Re(z-w-\theta \nu)}{4 Y V}
\end{aligned}
$$

$\left(Z, W \in \mathcal{H}^{(1 \mid 1)}, W=(w, \nu)=\left(u+i v, \nu_{1}+i \nu_{2}\right), V=v+i \nu \bar{\nu} / 2\right)$ as derived form classical mechanics on the Poincaré super upper half-plane [3, 48, 68]. We introduce the DiracLaplace operators $\square_{m}$ and $\widehat{\square}_{m}$, respectively $[3,5]$

$$
\square_{m}=2 Y D \bar{D}+i m(\bar{\theta}-\theta) \bar{D}, \quad \widehat{\square}_{m}=2 Y D \bar{D}+\frac{i m}{2}(\bar{\theta}-\theta)(D+\bar{D}),
$$

and $\square_{m}$ and $\widehat{\square}_{m}$ are related by a linear isomorphism $\square_{m}=Y^{-m / 2}\left(\widehat{\square}_{m}+i m / 2\right) Y^{m / 2}$. Particularly we have for $m=0$

$$
\widehat{\square}_{0}=\square_{0} \equiv \square=2 Y\left(\partial_{\theta} \partial_{\bar{\theta}}+\theta \bar{\theta} \partial_{z} \partial_{\bar{z}}+\theta \partial_{\bar{\theta}} \partial_{z}-\bar{\theta} \partial_{\theta} \partial_{\bar{z}}\right) .
$$

With the notation $-\Delta_{m}=-4 y^{2} \partial_{z} \partial_{\bar{z}}+i m y \partial_{x}=-y^{2}\left(\partial_{x}^{2}+\partial_{y}^{2}\right)+i m y \partial_{x}$ we obtain for a super function

$$
\Psi(Z, \bar{Z})=A(z, \bar{z})+\frac{\theta \bar{\theta}}{y} B(z, \bar{z})+\frac{1}{\sqrt{y}}(\theta \chi(z, \bar{z})+\bar{\theta} \widetilde{\chi}(z, \bar{z}))
$$

the following equivalence $[5,33,52]$

$$
\begin{aligned}
\hat{\square}_{m} \Psi(Z, \bar{Z})=s \Psi(Z, \bar{Z}) \\
\qquad\left\{\begin{array}{l}
-\Delta_{m} A(z, \bar{z})=s(s+i) A(z, \bar{z}), \\
B(z, \bar{z})=\frac{s}{2} A(z, \bar{z}), \\
\left(s-\frac{i m}{2}\right) \tilde{\chi}(z, \bar{z})=-2 y \partial_{\bar{z}} \chi(z, \bar{z})+\frac{i}{2}(m+1) \chi(z, \bar{z}) \\
-\Delta_{(m+1)} \chi(z, \bar{z})=\left(\frac{1}{4}+s^{2}\right) \chi(z, \bar{z}) .
\end{array}\right.
\end{aligned}
$$


An explicit solution of Eq.(3.16) for $m=0$ on the entire $\mathcal{H}^{(1 \mid 1)}$ is given by [48]

$$
\begin{aligned}
\Phi_{p, k}(z, \bar{z}, \theta, \bar{\theta})= & \sqrt{\frac{2 i \sinh \pi p}{\pi^{3}}}\left(1-i \frac{1+2 i p}{4 y} \theta \bar{\theta}\right) \sqrt{y} e^{i k x} K_{i p}(|k| y) \\
\phi_{p, k}(z, \bar{z}, \theta, \bar{\theta})= & \sqrt{\frac{\cos [\pi(c+i p)]}{2 \pi^{2}(c+i p)^{\sigma_{k}-1}}} \frac{e^{i k x}}{\sqrt{y}} \\
& \times\left[\theta W_{\sigma_{k} / 2, c+i p}(2|k| y)+i(c+i p)^{\sigma_{k}} \bar{\theta} W_{-\sigma_{k} / 2, c+i p}(2|k| y)\right]
\end{aligned}
$$

with $s=-i\left(\frac{1}{2}+i p\right), \sigma_{k}=\operatorname{sign}(k),(k \neq 0)$, and $c \in \mathbb{R},|c| \leq \frac{1}{2} . K_{\nu}$ and $W_{\mu, \nu}$ denote modified Bessel- and Whittaker-functions, respectively. Due to the particular form of the differential equation for $\Phi(Z, \bar{Z})$ we see that the solutions can be characterised by their parity with respect to the coordinate $x$, i.e. they can have even and odd parity with respect to $x$.

I have proposed in II similarly as for the hyperbolic $T \in \Gamma$, elliptic and parabolic $T \in \Gamma$, and appropriate super fundamental domains $\mathcal{F}^{(1 \mid 1)}$, a decomposition of an appropriate $T \in \Gamma$ as follows [34]

$$
\begin{aligned}
(T \in \Gamma \text { conjugate to }) \quad \gamma \times R \times S & =\left(\begin{array}{ccc}
N_{\gamma}^{1 / 2} & 0 & 0 \\
0 & N_{\gamma}^{-1 / 2} & 0 \\
0 & 0 & \chi_{\gamma}
\end{array}\right) \\
& \times\left(\begin{array}{ccc}
\cos \phi & -\sin \phi & 0 \\
\sin \phi & \cos \phi & 0 \\
0 & 0 & \chi_{R}
\end{array}\right) \cdot\left(\begin{array}{ccc}
1 & n & 0 \\
0 & 1 & 0 \\
0 & 0 & \chi_{S}
\end{array}\right),
\end{aligned}
$$

with $n \in \mathbb{N}$ and $0<\phi<\pi$, and $\gamma, R$ and $S$, respectively, denote hyperbolic, elliptic and parabolic transformations, acting by matrix multiplication [c.f. p.9]. The body $\mathcal{F}$ of a fundamental domain $\mathcal{F}^{(1 \mid 1)}$ has according to [39] $4 g+2 s+2 \kappa$ sides, the boundaries being geodesics, of course. We also maintain the notion of $\chi_{T}$ irrespective, whether $T \in \Gamma$ is hyperbolic, elliptic or parabolic, respectively, and we choose $\chi_{T}$ according to the spin structure of the super Riemann surface in question. For a super Riemann surface of genus $g$ there are obviously $2^{(\# g \text { enerators })}=2^{(2 g+s+\kappa)}$ possible spin structures.

The constraint (3.9) is altered due to the presence of of elliptic fixed points and cusps according to $[39,71]$

$$
\left(\gamma_{0} \gamma_{1}^{-1} \ldots \gamma_{2 g-2} \gamma_{2 g-1}^{-1}\right)\left(\gamma_{0}^{-1} \gamma_{1} \ldots \gamma_{2 g-2}^{-1} \gamma_{2 g-1}\right) R_{1} \ldots R_{s} S_{1} \ldots S_{\kappa}=\mathbb{1}_{2 \mid 1}
$$

3. Construction of bordered super Riemann surfaces. Because it is sufficient to consider super Riemann surfaces without odd parameters we can propose a construction of a bordered super Riemann surface. To construct a bordered super Riemann surface we take the construction of a usual bordered Riemann surface and endow it with the Grassmann algebra $\Lambda_{\infty}$. Because we know how to define a closed super Riemann surface, we take $\widehat{\Sigma}$ 
and enlarge it to $\widehat{\Sigma}^{(1 \mid 1)}$ together with its corresponding super Fuchsian group $\widehat{\Gamma}^{(1 \mid 1)}$ constructed from $\widehat{\Gamma}$ and the super fundamental domain $\widehat{\mathcal{F}}^{(1 \mid 1)}$. A convenient way to introduce the super-analogue of the involution $\mathcal{I}$ turns out to be the super involution

$$
\left.\begin{array}{l}
\mathcal{I} Z=\mathcal{I}(z, \theta)=(-\bar{z},-i \bar{\theta}), \\
\mathcal{I} \bar{Z}=\mathcal{I}(\bar{z}, \bar{\theta})=(-z,-i \theta)
\end{array}\right\}
$$

respectively $\mathcal{I}\left(z, \theta_{1}, \theta_{2}\right)=\left(-\bar{z},-i \theta_{1}, i \theta_{2}\right)$. It has the properties

$$
\mathcal{I} D=i \bar{D}, \quad \mathcal{I} \bar{D}=i D
$$

Note $\mathcal{I}^{4} Z=Z$ and $\mathcal{I}^{4} D=D$. Furthermore for the Dirac-Laplace operator $\widehat{\square}_{m}$ we have

$$
\mathcal{I} \widehat{\square}_{m}=\widehat{\square}_{-m}=\widehat{\widehat{\square}}_{m}
$$

Similarly as for the usual bordered Riemann surface where $\Sigma=\widehat{\Sigma} \backslash \mathcal{I}$, we then define the bordered super Riemann surface $\Sigma^{(1 \mid 1)}$ as $\Sigma^{(1 \mid 1)}=\widehat{\Sigma}^{(1 \mid 1)} \backslash \mathcal{I}$. The corresponding discs $d_{1}^{(1 \mid 1)}, \ldots, d_{n}^{(1 \mid 1)}$ then are super-conformal non-overlapping superdiscs seen as usual conformal non-overlapping discs endowed with the Grassmann algebra $\Lambda_{\infty}$. The particular form of the involution (3.21) enables us to work directly on the fundamental domains $\widehat{\mathcal{F}}^{(1 \mid 1)}$. The super Fuchsian group $\widehat{\Gamma}$ is consequently a symmetric super Fuchsian group.

4. The Selberg supertrace formula for hyperbolic conjugacy classes. Turning to the Selberg supertrace formula, let us introduce the Selberg super operator $L$ by $[4-7,33]$

$$
\begin{aligned}
(L \phi)(Z) & =\int_{\mathcal{H}^{(1 \mid 1)}} d V(W) k_{m}(Z, W) \phi(W), \\
k_{m}(Z, W) & =J^{m}(Z, W)\{\Phi[R(Z, W)]+r(Z, W) \Psi[R(Z, W)]\}, \\
J^{m}(Z, W) & =\left(\frac{z-\bar{w}-\theta \bar{\nu}}{\bar{z}-w-\bar{\theta} \nu}\right)^{m / 2} .
\end{aligned}
$$

$k_{m}(Z, W)$ is the integral kernel of an operator valued function of the Dirac-Laplace operator $\square_{m}$ (respectively $\hat{\square}_{m}$ ), and $\Phi$ and $\Psi$ are sufficiently decreasing functions at infinity. Note $J^{m}(\gamma Z, \gamma W)=j(\gamma, Z) J^{m}(Z, W) j^{-1}(\gamma, W)$ with $j(\gamma, Z)$ given by $j(\gamma, Z)=\left(F_{\gamma} /\left|F_{\gamma}\right|\right)^{m}$, where $F_{\gamma}=D \theta^{\prime}[33,52]$. We have $j(\gamma \sigma, Z)=j(\gamma, \sigma Z) j(\sigma, Z)\left(\forall \gamma, \sigma \in \Gamma\right.$ and $\left.Z \in \mathcal{H}^{(1 \mid 1)}\right)$. A superautomorphic form $f(Z)$ is then defined by $[4,33] f(\gamma Z)=j(\gamma, Z) f(Z)(\forall \gamma \in \Gamma)$. The super-automorphic kernel is defined as

$$
K(Z, W)=\frac{1}{2} \sum_{\{\gamma\}} k_{m}(Z, \gamma W) j(\gamma, W)
$$

(" $\frac{1}{2}$ " because both $\gamma$ and $-\gamma$ have to be included in the sum) i.e. $(L \phi)(z)=\left[h\left(\square_{m}\right)\right](z)$. $L$ is acting on super-automorphic functions $f(Z)$. 
For the point pair invariants we find for the action of $\mathcal{I}$

$$
\left.\begin{array}{rl}
R(Z, \mathcal{I} W) & =R(\mathcal{I} Z, W) \\
r(Z, \mathcal{I} W) & =\overline{r(\mathcal{I} Z, W)},
\end{array}\right\}
$$

furthermore $J(Z, \mathcal{I} W)=\overline{J(\mathcal{I} Z, W)}$, and due to the construction of $k_{m}$

$$
k_{m}(Z, \mathcal{I} W)=\overline{k_{m}(\mathcal{I} Z, W)} .
$$

Let $f$ be a super-automorphic function with $f(\gamma Z)=j(\gamma, Z) f(Z)$ and $g=L f$. Let $\mathcal{F}^{(1 \mid 1)}(\gamma)$ a fundamental domain of $\gamma \in \Gamma$ whose body equals $\mathcal{F}_{\text {red }}^{(1 \mid 1)}=\mathcal{F}$ (and is constructed in the same sense as the generalization $\mathcal{H}^{(1 \mid 1)}$ of $\mathcal{H}$ ). The expansion into hyperbolic conjugacy classes yields

$$
\begin{aligned}
\operatorname{str}(L) & =\int_{\mathcal{F}^{(1 \mid 1)}(\gamma)} d V(Z) K(Z, Z) \\
& =\int_{\mathcal{F}^{(1 \mid 1)}(\gamma)} \sum_{\gamma \in \Gamma} k_{m}(Z, \gamma Z) d V(Z)=\frac{i^{m}}{2} \mathcal{A} \Phi(0)+\sum_{\substack{\{\gamma\} \\
\operatorname{str}(\gamma)+\chi_{\gamma}>2}} \chi_{\gamma}^{m} A(\gamma) .
\end{aligned}
$$

Here I have assumed without loss of generality $a+d \geq 0$ for a $\gamma \in \widehat{\Gamma}$, since Aut $\mathcal{H}^{(1 \mid 1)}=$ $\operatorname{OSp}(2 \mid 1, \mathbb{R}) /\{ \pm \mathbb{1}\}$. The first term corresponds to the identity transformation (zero-length term) and the second $A(\gamma)$ is given by

$$
A(\gamma)=\chi_{\gamma}^{-m} \int_{\mathcal{F}^{(1 \mid 1)}(\gamma)} k_{m}(Z, \gamma Z) j(\gamma, W) d V(Z)
$$

In Refs. $[5,33]$ these two terms corresponding to the identity transformation and hyperbolic conjugacy classes, respectively, were calculated, i.e. I have discussed in detail

Theorem 3.1 [4-7, 33]: The Selberg supertrace formula for m-weighted Dirac-Laplace operators on closed super Riemann surfaces for hyperbolic conjugacy classes is given by:

$$
\begin{aligned}
& \sum_{n=0}^{\infty}\left[h\left(\frac{1+m}{2}+i p_{n}^{(B)}\right)-h\left(\frac{1+m}{2}+i p_{n}^{(F)}\right)\right]=-\frac{\mathcal{A}(\mathcal{F})}{4 \pi} \int_{0}^{\infty} \frac{g(u)-g(-u)}{\sinh \frac{u}{2}} \cosh \left(\frac{u m}{2}\right) d u \\
& \quad+\sum_{\{\gamma\}} \sum_{k=1}^{\infty} \frac{l_{\gamma} \chi_{\gamma}^{m k}}{2 \sinh \frac{k l_{\gamma}}{2}}\left[g\left(k l_{\gamma}\right)+g\left(-k l_{\gamma}\right)-\chi_{\gamma}^{k}\left(g\left(k l_{\gamma}\right) e^{-k l_{\gamma} / 2}+g\left(-k l_{\gamma}\right) e^{k l_{\gamma} / 2}\right)\right] .
\end{aligned}
$$

The test function $h$ is required to have the following properties

i) $h\left(\frac{1+m}{2}+i p\right) \in C^{\infty}(\mathbb{R})$,

ii) $h\left(\frac{1+m}{2}+i p\right)$ need not to be an even function in $p$,

iii) $h(p)$ vanishes faster than $1 /|p|$ for $p \rightarrow \pm \infty$.

iv) $h\left(\frac{1+m}{2}+i p\right)$ is holomorphic in the strip $\Im(p) \leq 1+\frac{m}{2}+\epsilon, \epsilon>0$, to guarantee absolute convergence in the summation over $\{\gamma\}$. 
The above Selberg supertrace formula (3.30) is valid for discrete hyperbolic conjugacy classes and in this case the noneuclidean area of the ("bosonic") fundamental domain is $\mathcal{A}=4 \pi(g-1)$. The Fourier transformation $g$ of $h$ is given by

$$
\begin{aligned}
& g(u)=\frac{1}{2 \pi} \int_{-\infty}^{\infty} h\left(\frac{1+m}{2}+i p\right) e^{-i u p} d p \\
&=\frac{1}{4} \int_{4 \sinh ^{2} \frac{u}{2}}^{\infty} \frac{d x}{(x+4)^{m / 2}}\left\{\frac{\Psi(x)+2\left(e^{u}-1\right) \Phi^{\prime}(x)}{\sqrt{x-4 \sinh ^{2} \frac{u}{2}}}\left[\alpha_{+}^{m}(x, u)+\alpha_{-}^{m}(x, u)\right]\right. \\
&\left.-i m e^{u / 2} \Phi(x) \frac{\alpha_{+}^{m}(x, u)-\alpha_{-}^{m}(x, u)}{x+4}\right\},
\end{aligned}
$$

where $\alpha_{ \pm}^{m}(x, u)=\left( \pm \sqrt{x-4 \sinh ^{2} \frac{u}{2}}-2 i \cosh \frac{u}{2}\right)^{m / 2}$. Specific trace formulæ, in particular for the heat kernel were considered by Aoki [3], Oshima [54], Yasui [47, 48] and Uehara and Yasui [68], as well as an explicit evaluation for the energy dependent resolvent kernel for the operator $\widehat{\square}^{2}[3,54]$. From Eq.(3.31) an explicit formula for $\Phi(x)$ can be derived [33] which has the form

$$
i^{m} \Phi(x)=\frac{1}{\pi \sqrt{x+4}} \int_{x}^{\infty} \frac{d y}{\sqrt{y+4}} \int_{-\infty}^{\infty} Q_{1}^{\prime}\left(y+t^{2}\right)\left(\frac{\sqrt{y+t^{2}+4}-t}{\sqrt{y+t^{2}+4}+t}\right)^{m / 2} d t,
$$

with $Q_{1}(w)=2 \operatorname{coth} \frac{u}{2}[g(u)-g(-u)], w=4 \sinh ^{2} \frac{u}{2}$. Let us consider the combination

$$
\begin{aligned}
& g(u) e^{-u / 2}-g(-u) e^{u / 2}=\frac{i^{m}}{2} \sinh \frac{u}{2} \\
& \times \int_{-\infty}^{\infty} d \xi\left(\frac{\sqrt{w+4}+i \xi}{\sqrt{w+4}-i \xi}\right)^{m / 2}\left[4 \Phi^{\prime}\left(w+\xi^{2}\right)-\Psi\left(w+\xi^{2}\right)\right] .
\end{aligned}
$$

We define $Q_{3}(w)=2\left[g(u) e^{-u / 2}-g(-u) e^{u / 2}\right] / \sinh \frac{u}{2}$ and obtain the general inversion formula for $\Psi(x)$

$$
i^{m} \Psi(x)=4 i^{m} \Phi^{\prime}(x)+\frac{1}{\pi} \int_{-\infty}^{\infty} Q_{3}^{\prime}\left(x+t^{2}\right)\left(\frac{\sqrt{x+4+t^{2}}-t}{\sqrt{x+4+t^{2}}-t}\right)^{m / 2} d t .
$$

Alternatively, this can be rewritten as

$$
i^{m} \Psi(x)=-\frac{i^{m} \Phi(x)}{2(x+4)}+\frac{1}{\pi} \int_{-\infty}^{\infty}\left(\frac{\sqrt{x+4+t^{2}}-t}{\sqrt{x+4+t^{2}}-t}\right)^{m / 2}\left[Q_{3}^{\prime}\left(x+t^{2}\right)-\frac{Q_{1}^{\prime}\left(x+t^{2}\right)}{x+4}\right] d t .
$$

For $m=0$ we obtain simple inversion formulæ for $\Phi(t)$ and $\Psi(t)$, respectively

$$
\Phi(t)=-\frac{1}{\pi} \int_{t}^{\infty} \frac{Q_{1}(w) d w}{(w+4) \sqrt{w-t}}, \quad \Psi(t)=-\frac{1}{2 \pi} \int_{t}^{\infty} \frac{Q_{2}^{\prime}(w) d w}{\sqrt{w-t}},
$$

with $Q_{2}(w)=2\left[g(u) e^{-u / 2}+g(-u) e^{u / 2}\right] / \cosh \frac{u}{2}$. The incorporation of the elliptic and parabolic conjugacy classes was discussed in Ref.[34] and is not repeated here. 


\section{The Selberg Supertrace Formula for Bordered Super Riemann Surfaces}

I first proceed by considering the Selberg supertrace formula where the body of the underlying fundamental domain is compact, and second where it is non-compact. Let us consider the super-automorphic Selberg operator with Dirichlet boundary-conditions

$$
\begin{aligned}
(\widehat{L} f)(Z) & =\frac{1}{4} \int_{\mathcal{H}^{(1 \mid 1)}} d V(W)\left[k_{m}(Z, W)-k_{m}(Z, \mathcal{I} W)\right] f(W) \\
& =\frac{1}{4} \sum_{\{\gamma\}} \int_{\widehat{\mathcal{F}}^{(1 \mid 1)}(\gamma)} d V(W)\left[k_{m}(Z, W)-k_{m}(Z, \mathcal{I} W)\right] f(W) \\
& =\frac{1}{2} \int_{\widehat{\mathcal{F}}^{(1 \mid 1)}(\gamma)} d V(W) K(Z, W) f(W),
\end{aligned}
$$

where

$$
K(Z, W)=\frac{1}{2} \sum_{\{\gamma\}}\left[k_{m}(Z, \gamma W)-k_{m}(Z, \gamma \mathcal{I} W)\right]
$$

is the super-automorphic kernel on bordered super Riemann surfaces. Now we have for a superfunction $\phi$ which is odd with respect to $x$

$$
\begin{aligned}
\frac{1}{2} & \int_{\widehat{\mathcal{F}}^{(1 \mid 1)}(\gamma)} d V(W) K(Z, \mathcal{I} W) \phi(W) \\
& =\frac{1}{4} \sum_{\{\gamma\}} \int_{\widehat{\mathcal{F}}^{(1 \mid 1)}(\gamma)} d V(W) k_{m}(Z, \gamma \mathcal{I} W) \phi(W) \\
& =-\frac{1}{4} \sum_{\{\gamma\}} \int_{\widehat{\mathcal{F}}^{(1 \mid 1)}(\gamma)} d V(\mathcal{I} W) k_{m}(Z, \gamma W) \phi(W) \\
& =-\frac{1}{4} \sum_{\{\gamma\}} \int_{\widehat{\gamma}^{(1 \mid 1)}(\gamma)} d V(\mathcal{I} W) k_{m}(Z, W) \phi(W) \\
& =\frac{1}{2} \int_{\mathcal{H}^{(1 \mid 1)}} d V(W) k_{m}(Z, \mathcal{I} W) \phi(W) \\
& =\frac{1}{2} \int_{\mathcal{H}^{(1 \mid 1)}} d V(W) \overline{k(\mathcal{I} Z, W)} \phi(W)=\frac{1}{2} \overline{(L \bar{\phi})(\mathcal{I} Z)},
\end{aligned}
$$

due to the properties of the super Selberg operator. Let now $\Phi$ be an eigenfunction of $\widehat{\square}_{m}$ which is odd with respect to $x$, i.e. $\widehat{\square}_{m} \Phi=s \Phi$. Then $\overline{s \Phi}=\bar{s} \bar{\Phi}=\widehat{\square}_{-m} \bar{\Phi}$ and $\bar{\Phi}$ is an odd eigenfunction of $\hat{\square}_{-m}$ with eigenvalue $\bar{s}$. Denote by $\widehat{L}$ the Selberg super operator on the super Riemann surface $\widehat{\Sigma}$; let $(L \phi)(Z)=\Lambda(s) \phi(Z)$ and $\overline{(L \bar{\phi})(\mathcal{I} Z)}=\overline{\Lambda^{\prime}(\bar{s})} \phi(\mathcal{I} Z)$ on $\Sigma$ and $\mathcal{I} \Sigma$, respectively. Then

$$
\begin{aligned}
(\widehat{L} \phi)(Z) & =\frac{1}{2}(L \phi)(Z)-\frac{1}{2} \overline{(L \bar{\phi})(\mathcal{I} Z)} \\
& =\frac{1}{2} \Lambda(s) \phi(Z)-\frac{1}{2} \overline{\Lambda^{\prime}(\bar{s})} \phi(\mathcal{I} Z)=\frac{1}{2}\left[\Lambda(s)+\Lambda^{\prime}(s)\right] \phi(Z) .
\end{aligned}
$$


The equivalence relation (3.16) shows that the eigenvalue problem for the operator $\hat{\square}_{m}$ is closely related to the eigenvalue problem of the operator $-\Delta_{m}$, both for eigenfunctions which are even or odd with respect to $x$. Now, an odd eigenfunction of $-\Delta_{m}$ is also an odd eigenfunction of $-\Delta_{-m}$, and the solution of the corresponding differential equations depend only on $m^{2}$ but not on $m$ [38, pp.266-68; 25, pp.203-5], hence, the spectrum depends only on $|m|$ (compare also [12]). Therefore we conclude that a with-respect-to$x$ odd eigenfunction of $\square_{m}$ is also a with-respect-to- $x$ odd eigenfunction of $\mathcal{I} \square_{m}$ with the eigenvalue $\bar{s}$, furthermore $\Lambda=\Lambda^{\prime}$ [12], and we can infer [together with the usual identification $\left.h(p)=\Lambda\left(\frac{1}{2}+i p\right)\right]$

$$
(\widehat{L} \phi)(Z)=h(p) \phi(z)
$$

Let $Z_{\Gamma}(\gamma)$ the centralizer of a $\gamma \in \Gamma$. For $\operatorname{str}(\widehat{L})$ we obtain on the one hand

$$
\operatorname{str}(\widehat{L})=\sum_{n}\left[h\left(p_{n}^{(B)}\right)-h\left(p_{n}^{(F)}\right)\right]
$$

where $s_{n}^{(B, F)}=\frac{1}{2}+i p_{n}^{(B, F)}$ are the bosonic and fermionic eigenvalues, respectively, of $\square_{m}$. [According to Eq.(3.16) we should consequently write $s=-i\left(\frac{1}{2}+i p\right)$, which looks, however, somewhat artificial and is therefore not adopted.] On the other we have

$$
\begin{aligned}
\operatorname{str}(\widehat{L}) & =\frac{1}{2} \int_{\widehat{\mathcal{F}}^{(1 \mid 1)}(\gamma)} d V(W) K(Z, Z) \\
& =\frac{1}{4} \sum_{\{\gamma\}} \int_{\widehat{\mathcal{F}}^{(1 \mid 1)}(\gamma)}\left[k_{m}(Z, \gamma Z)-k_{m}(Z, \gamma \mathcal{I} Z)\right] d V(Z),
\end{aligned}
$$

where $\widehat{\mathcal{F}}^{(1 \mid 1)}(\gamma)$ denotes the fundamental region for the super Fuchsian group $Z_{\Gamma}(\gamma)$, the centralizer of $\gamma \in \Gamma$.

1) $\widehat{\mathcal{F}}_{\text {red }}^{(1 \mid 1)}$ is compact. For convenience we set $\rho=\gamma \mathcal{I}$ and use the classification of the inversehyperbolic transformations according to $\rho \in \bar{\Gamma} \mathcal{I}$, respectively, $\rho^{2} \in \bar{\Gamma}$. We generalize the result of the conjugacy classes for the usual case of bordered Riemann surfaces and consider the two cases i) and ii) for the conjugacy classes in $\gamma \mathcal{I}$ (c.f. p.6). The expansion into the conjugacy classes yields for the Selberg super operator for Dirichlet boundary-conditions [c.f. the discussion following Eq.(2.12)]

$$
\begin{aligned}
\operatorname{str}(\widehat{L}) & =\frac{1}{2} \int_{\widehat{\mathcal{F}}^{(1 \mid 1)}(\gamma)} \sum_{\{\gamma\}}\left[k_{m}(Z, \gamma Z)-k_{m}(Z, \gamma \mathcal{I} Z)\right] d V(Z) \\
& =\frac{\widehat{\mathcal{A}}}{4} \Phi(0)+\frac{1}{2} \sum_{\{\gamma\}} \int_{\widehat{\mathcal{F}}^{(1 \mid 1)}(\gamma)} k_{m}(Z, \gamma Z)-\frac{1}{2} \sum_{\{\rho\} ; \widehat{\Gamma}_{\rho, h y p}} \int_{\widehat{\mathcal{F}}^{(1 \mid 1)}(\rho)} k_{m}(Z, \rho Z) .
\end{aligned}
$$


Let us consider the involution term. We obtain

$$
\begin{aligned}
& \left.\sum_{\gamma \in \bar{\Gamma}} \int_{\widehat{\mathcal{F}}^{(1 \mid 1)}(\gamma)} d V(Z) k_{m}[Z, \mathcal{I} Z)\right] \\
& \quad=\sum_{\rho \in \bar{\Gamma} \mathcal{I}} \int_{\widehat{\mathcal{F}}^{(1 \mid 1)}(\gamma)} d V(Z) k_{m}(Z, \rho Z)=: \sum_{\rho \in \bar{\Gamma} \mathcal{I}} A(\rho) \\
& \quad=\sum_{\rho_{p}} \sum_{k=0}^{\infty} A\left(\rho_{p}^{2 k+1}\right)+\sum_{i=1}^{n} \sum_{\rho_{i}} \sum_{k=0}^{\infty} A\left(\rho_{i}^{2 k+1}\right) .
\end{aligned}
$$

Now observe

$$
k_{m}\left(\gamma Z, \rho^{2 k+1} \gamma Z\right)=k_{m}\left(Z, \gamma^{-1} \rho^{2 k+1} \gamma Z\right) j(\gamma, Z) j^{-1}\left(\gamma, \gamma^{-1} \rho^{2 k+1} \gamma Z\right),
$$

and

$$
\begin{aligned}
j\left(\rho^{2 k+1} \gamma, Z\right) & =j(\gamma, Z) j\left(\rho^{2 k+1}, \gamma Z\right)=j\left(\gamma \cdot \gamma^{-1} \rho^{2 k+1} \gamma, Z\right) \\
& =j\left(\gamma, \gamma^{-1} \rho^{2 k+1} \gamma Z\right) j\left(\gamma^{-1} \rho^{2 k+1} \gamma, Z\right) .
\end{aligned}
$$

We then get

$$
\begin{aligned}
& \sum_{\rho} \sum_{k=0}^{\infty} A\left(\rho^{2 k+1}\right)=\sum_{\{\rho\}} \sum_{\sigma \in\{\rho\}} \sum_{k=0}^{\infty} \int_{\widehat{\mathcal{F}}^{(1 \mid 1)}} d V(Z) k_{m}\left(Z, \sigma^{2 k+1} Z\right) \\
& =\sum_{\{\rho\}} \sum_{\gamma \in \Gamma_{\rho^{2} \backslash \bar{\Gamma}}} \sum_{k=0}^{\infty} \int_{\widehat{\mathcal{F}}^{(1 \mid 1)}} d V(Z) k_{m}\left(Z, \gamma^{-1} \rho^{2 k+1} \gamma Z\right) \\
& =\sum_{\{\rho\}} \sum_{\gamma \in \Gamma_{\rho^{2}} \backslash \bar{\Gamma}} \sum_{k=0}^{\infty} \int_{\widehat{\mathcal{F}}^{(1 \mid 1)}} d V(Z) j\left(\rho^{2 k+1}, \gamma Z\right) k\left(\gamma Z, \rho^{2 k+1} \gamma Z\right) \\
& =\sum_{\{\rho\}} \sum_{k=0}^{\infty} \sum_{\gamma \in \Gamma_{\rho^{2} \backslash \bar{\Gamma}}} j\left(\rho^{2 k+1}, Z\right) \int_{\gamma \widehat{\mathcal{F}}^{(1 \mid 1)}} d V(Z) k_{m}\left(Z, \rho^{2 k+1} Z\right) \\
& =\sum_{\{\rho\}} \sum_{k=0}^{\infty} \int_{\Gamma_{\rho^{2}} \backslash \mathcal{H}} d V(Z) j\left(\rho^{2 k+1}, Z\right) k_{m}\left(Z, \rho^{2 k+1} Z\right) .
\end{aligned}
$$

By an overall conjugation in $\operatorname{OSp}(2 \mid 1, \mathbb{R})$ we can arrange for $\gamma$ to be a dilatation, i.e. $\rho z=-\sqrt{N} \bar{z}$ and $\nu_{1}=\rho \theta_{1}=-\chi_{\rho} N^{1 / 4} \theta_{1}, \nu_{2}=\rho \theta_{2}=-\chi_{\rho} N^{1 / 4} \theta_{2}$. Similarly as in the usual hyperbolic case [33] we find for the two-point invariants $\left[M=N^{k+1 / 2}\right]$

$$
\begin{aligned}
R(Z, \rho Z) & =\frac{|z+M \bar{z}|^{2}}{M y^{2}}\left(1-\frac{2 \theta_{1} \theta_{2}}{y}\right) \equiv R_{0}\left(1-\frac{2 \theta_{1} \theta_{2}}{y}\right) \\
r(Z, \rho Z) & =\frac{\theta_{1} \theta_{2}}{y}\left[2+\chi\left(M^{1 / 2}+M^{-1 / 2}\right)\right] .
\end{aligned}
$$


Furthermore $j\left(\rho^{2 k+1}, Z\right)=\chi_{\rho}^{(2 k+1) m}$ and

$$
J^{m}\left(Z, \rho^{2 k+1} Z\right)=\left(\frac{\zeta+2 i \cosh \frac{u}{2}}{\zeta-2 i \cosh \frac{u}{2}}\right)^{m / 2}\left(1-\frac{2 i m \chi_{\rho}^{2 k+1} \zeta \theta_{1} \theta_{2}}{y\left(\zeta^{2}+4 \cosh ^{2} \frac{u}{2}\right)}\right)
$$

where $\zeta=2 x \cosh \frac{u}{2} / y$ and $u=(2 k+1) \ln \sqrt{M}=(k+1 / 2) l_{\rho^{2}}$. The evaluation of the conjugacy classes $\{\rho\}$ is straightforward and similar to the usual hyperbolic case. Evaluating the relevant terms we obtain

Theorem 4.1: The Selberg supertrace formula for $m$-weighted Dirac-Laplace operators $\square_{m}$ on compact bordered super Riemann surfaces with Dirichlet boundary-conditions is given by:

$$
\begin{aligned}
& \sum_{n=1}^{\infty}\left[h\left(p_{n}^{(B)}\right)-h\left(p_{n}^{(F)}\right)\right]=-\frac{\widehat{\mathcal{A}}}{4 \pi} \int_{0}^{\infty} \frac{g(u)-g(-u)}{\sinh \frac{u}{2}} \cosh \left(\frac{u m}{2}\right) d u \\
& +\frac{1}{4} \sum_{\{\gamma\}} \sum_{k=1}^{\infty} \frac{\chi_{\gamma}^{k m} l_{\gamma}}{\sinh \frac{k l_{\gamma}}{2}}\left[g\left(k l_{\gamma}\right)+g\left(-k l_{\gamma}\right)-\chi_{\gamma}^{k}\left(g\left(k l_{\gamma}\right) e^{-k l_{\gamma} / 2}+g\left(-k l_{\gamma} / 2\right) e^{k l_{\gamma} / 2}\right)\right] \\
& -\frac{1}{4} \sum_{\left\{\rho^{2}\right\}} \sum_{k=0}^{\infty} \frac{\chi_{\rho^{2}}^{(k+1 / 2) m} l_{\rho^{2}}}{\cosh \left[\frac{1}{2}\left(k+\frac{1}{2}\right) l_{\rho^{2}}\right]}\left\{g\left[\left(k+\frac{1}{2}\right) l_{\rho^{2}}\right]+g\left[-\left(k+\frac{1}{2}\right) l_{\rho^{2}}\right]\right. \\
& -\quad \chi_{\rho^{2}}^{k+\frac{1}{2}}\left(g\left[\left(k+\frac{1}{2}\right) l_{\rho^{2}}\right] e^{\left.\left.-\frac{1}{2}\left(k+\frac{1}{2}\right) l_{\rho^{2}}+g\left[-\left(k+\frac{1}{2}\right) l_{\rho^{2}}\right] e^{\frac{1}{2}\left(k+\frac{1}{2}\right) l_{\rho^{2}}}\right)\right\}}\right. \\
& -\frac{1}{2} \sum_{i=1}^{n} \sum_{k=1}^{\infty} \frac{\chi_{C_{i}}^{k m} l_{C_{i}}}{\cosh \frac{k l_{C_{i}}}{2}}\left[g\left(k l_{C_{i}}\right)+g\left(-k l_{C_{i}}\right)-\chi_{C_{i}}^{k}\left(g\left(k l_{C_{i}}\right) e^{-k l_{C_{i}} / 2}+g\left(-k l_{C_{i}}\right) e^{k l_{C_{i}} / 2}\right)\right],
\end{aligned}
$$

where $\lambda_{n}^{(B, F)}=\frac{1}{2}+i p_{n}^{(B, F)}$ on the left runs through the set of all eigenvalues of this Dirichlet problem, and the summation on the right is taken over all primitive conjugacy classes $\{\gamma\}_{\widehat{\Gamma}}, \operatorname{str}(\gamma)+\chi_{\gamma}>2$, and $\{\rho\}_{\widehat{\Gamma}}, \rho$ hyperbolic.

The test function $h$ is required to have the following properties

i) $h(p) \equiv h\left(\frac{1+m}{2}+i p\right) \in C^{\infty}(\mathbb{R})$,

ii) $h(p)$ need not to be an even function in $p$,

iii) $h(p)$ vanishes faster than $1 /|p|$ for $p \rightarrow \pm \infty$.

iv) $h(p)$ is holomorphic in the strip $\Im(p) \leq 1+\frac{m}{2}+\epsilon, \epsilon>0$, to guarantee absolute convergence in the summation over $\{\gamma\}$ and $\{\rho\}$.

Note that there is no $k=0$ contribution from the last summand. $g(u)$ is given by Eq.(3.31).

Note that in the case of Neumann boundary-conditions the last two terms just change their signs. 
2) $\widehat{\mathcal{F}}_{\text {red }}^{(1 \mid 1)}$ is non-compact. I only consider the case $m=0$. We now include all relevant conjugacy classes and get

$$
\begin{aligned}
\operatorname{str}(\widehat{L})= & \frac{1}{2} \int_{\widehat{\mathcal{F}}^{(1 \mid 1)}(T)} \sum_{\{T\}}[k(Z, T Z)-k(Z, T \mathcal{I} Z)] d V(Z) \\
= & \frac{1}{4} \widehat{\mathcal{A}} \Phi(0)+\frac{1}{2} \sum_{\substack{\{\gamma\} \\
\operatorname{str}(\gamma)+\chi_{\gamma}>2}} \int_{\widehat{\mathcal{F}}^{(1 \mid 1)}(\gamma)} k(Z, \gamma Z) \\
& -\frac{1}{2} \sum_{\{\rho\} ; \widehat{\Gamma}_{\rho, h y p}} \int_{\widehat{\mathcal{F}}^{(1 \mid 1)}(\rho)} k(Z, \rho Z) \\
& +\frac{1}{2} \sum_{\operatorname{str}(R)+\widehat{\Gamma}_{R}<2} \int_{\widehat{\mathcal{F}}(1 \mid 1)(R)} k(Z, R Z) \\
& +\frac{1}{2} \lim _{y_{m} \rightarrow \infty} \int_{\widehat{\mathcal{F}}_{y_{M}}^{(1 \mid 1)}} d V(Z) \\
& \times\left\{\sum_{\{S\}} \sum_{\gamma^{\prime} \in \widehat{\Gamma}_{S} \backslash \Gamma} k\left(Z, \gamma^{\prime-1} S \gamma^{\prime} Z\right)-\sum_{\substack{\{\rho\} ; \widehat{\Gamma}_{\rho, e l l} \\
\operatorname{str}(\rho)+\chi_{\rho}=0}} \sum_{\gamma^{\prime} \in \widehat{\Gamma}_{S} \backslash \Gamma} k\left(Z, \gamma^{\prime-1} \rho \gamma^{\prime} Z\right)\right\}
\end{aligned}
$$

with some properly defined compact domain $\widehat{\mathcal{F}}_{y_{M}}^{(1 \mid 1)}$ depending on a large parameter $y_{M}$, and where the sum is taken over all hyperbolic conjugacy classes $\{\gamma\}$, elliptic conjugacy classes $\{R\}$ and parabolic conjugacy classes $\{S\}$ in $\bar{\Gamma}$ with representatives $\gamma, R$ and $S$, respectively, over all relative non-degenerate classes $\{\rho\}, \rho$ hyperbolic, and over the relative conjugacy classes $\{\rho\}$ with $\operatorname{str}(\rho)+\chi_{\rho}=0, \rho$ elliptic.

The hyperbolic contributions have just been calculated (c.f. Eq.(4.16). Because there are no additional elliptic terms, we can just take the result of Ref.[34] [c.f. the discussion following Eq.(2.12)] and obtain

$$
\begin{aligned}
& \frac{1}{4} \int_{\widehat{\mathcal{F}}^{(1 \mid 1)}(R)} k(Z, R Z)=\frac{1}{2} \sum_{\{R\}} \sum_{k=1}^{\nu-1} \frac{1}{\nu}\left\{\left(1-\chi_{R}^{k} \cos \frac{k \pi}{\nu}\right)\right. \\
& \left.\times \int_{0}^{\infty} \frac{g(u) e^{-u / 2}+g(-u) e^{u / 2}}{\cosh u-\cos (2 k \pi / \nu)} d u+\int_{0}^{\infty} \frac{g(u)-g(-u)}{\cosh u-\cos (2 k \pi / \nu)} \sinh \frac{u}{2} d u\right\} .
\end{aligned}
$$


Turning to the "parabolic terms" we consider the transformation $Z \rightarrow W=\mathcal{I} S^{n} Z$. In Ref.[34] I obtained by considering $y_{M}$ finite with the corresponding fundamental domain $\widehat{\mathcal{F}}_{y_{M}}^{(1 \mid 1)}(S)$

$$
\begin{aligned}
\frac{1}{2} \int_{\widehat{\mathcal{F}}_{y_{M}}^{(1 \mid 1)}(S)} \frac{d \theta d \bar{\theta}}{Y} k(Z, S Z) \\
=\frac{1}{2} \int_{0}^{1} d x \int_{0}^{y_{M}} d y \int \frac{d \theta d \bar{\theta}}{Y} \sum_{n \neq 0} k\left(Z, S^{n} Z\right) \\
=\kappa_{-}\left\{\left(\ln y_{M}-\ln 2\right) g(0)+\frac{1}{2} \int_{0}^{\infty} g(-u) d u\right. \\
\left.-\frac{1}{4 \pi} \int_{-\infty}^{\infty}[\Psi(1-i p)+\Psi(1+i p)] h(p) d p\right\}+\frac{\kappa}{4} \int_{0}^{\infty}[g(u)-g(-u)] d u+O\left(\frac{1}{\sqrt{y_{M}}}\right) \\
=\kappa_{-}\left[\left(\ln y_{M}+C-\ln 2\right) g(0)+\frac{1}{2} \int_{0}^{\infty} g(-u) d u\right. \\
\left.\quad-\frac{1}{2} \int_{0}^{\infty} \ln \left(1-e^{-u}\right)\left\{\frac{d}{d u}[g(u)+g(-u)]\right\} d u\right]+\frac{\kappa}{4} \int_{0}^{\infty}[g(u)-g(-u)] d u+O\left(\frac{1}{\sqrt{y_{M}}}\right)
\end{aligned}
$$

$\left[\kappa_{ \pm}=\sum_{\{S\}}\left(1 \pm \chi_{S}\right)\right]$, and I have stated the result in two alternative ways. Note that Euler's constant $C=0.57721566490 \ldots$ appears only in the representation where $g(u)$ instead of $h(p)$ is used.

As we know from the discussion in section III from the usual Selberg case, the conjugacy class with $\operatorname{tr}(\rho)=0, \rho$ elliptic, contains an element of order two. In the super-case this is generalized to

$$
\gamma_{a}=\left(\begin{array}{ccc}
0 & a & 0 \\
-a^{-1} & 0 & 0 \\
0 & 0 & \chi_{\gamma_{a}}
\end{array}\right), \quad(\bmod \pm 1)
$$

with some $a \geq 1$. Because $\gamma_{a}$ is an elliptic element of order two we have to consider

$$
\int_{\bigcup \gamma^{\prime} \widehat{\mathcal{F}}_{y_{M}}^{(1 \mid 1)}, \gamma^{\prime} \in \widehat{\Gamma}} k(Z, \rho Z)=|\widehat{\Gamma}(\rho)| \int_{\bigcup \gamma^{\prime} \widehat{\mathcal{F}}_{y_{M}}^{(1 \mid 1)}, \gamma^{\prime} \in \widehat{\Gamma} \mathcal{I} \backslash \widehat{\Gamma}} k(Z, \rho Z)
$$

and $|\widehat{\Gamma}(\rho)|=\operatorname{order}[\widehat{\Gamma}(\rho)]=2$ which yields an additional factor $\frac{1}{2}$ in the second term in the parabolic contribution of Eq.(4.17).

For a proper asymptotic expansion [71] of the corresponding integral we remove from $\mathcal{H}^{(1 \mid 1)}$ two regions, denoted by $B_{1}^{(1 \mid 1)}=\left\{Z \in \mathcal{H}^{(1 \mid 1)} \mid x \geq y_{M}\right\}$ and $B_{2}^{(1 \mid 1)}=\gamma_{a} B_{1}$, respectively, i.e. we consider

$$
B^{(1 \mid 1)}=\mathcal{H}^{(1 \mid 1)}-B_{1}^{(1 \mid 1)}-B_{2}^{(1 \mid 1)}
$$


First let us insert a $n=0$ "parabolic term" into the super-automorphic kernel; this gives the integral

$$
\bigcup_{\bigcup \widehat{\mathcal{F}}_{y_{M}}^{(1 \mid 1)},\{\gamma\}} k(Z, \rho Z)=\int_{B^{(1 \mid 1)}} d V(Z) k(Z, \mathcal{I} Z)+o(1), \quad\left(y_{m} \rightarrow \infty\right),
$$

whose asymptotic behaviour must be studied. By the definition of $B^{(1 \mid 1)}$ the above integral separates into two contributions

$$
\int_{0}^{\infty} d x \int_{a^{2} / y_{M}}^{y_{M}} d y \int \frac{d \theta_{1} d \theta_{2}}{y+\theta_{1} \theta_{2}} k(Z, \mathcal{I} Z)-\int_{0}^{a^{2} / y_{M}} d y \int_{y \sqrt{a^{2} / y y_{M}-1}}^{\infty} d x \int \frac{d \theta_{1} d \theta_{2}}{y+\theta_{1} \theta_{2}} k(Z, \mathcal{I} Z)
$$

In order to evaluate the first integral we set $y_{0}=a^{2} / y_{M}$.

$$
\begin{aligned}
\frac{1}{2} \int_{0}^{\infty} & d x \int_{y_{0}}^{y_{M}} d y \int \frac{d \theta_{1} d \theta_{2}}{y+\theta_{1} \theta_{2}}\{\Phi[R(Z, \mathcal{I} Z)]+r(Z, \mathcal{I} Z) \Psi[R(Z, \mathcal{I} Z)]\} \\
& =\int_{0}^{\infty} d x \int_{y_{0}}^{y_{M}} \frac{d y}{y^{2}}\left[\frac{1}{2} \Phi\left(\frac{4 x^{2}}{y^{2}}\right)+\frac{4 x^{2}}{y^{2}} \Phi^{\prime}\left(\frac{4 x^{2}}{y^{2}}\right)+\left(1-\chi_{S}\right) \Psi\left(\frac{4 x^{2}}{y^{2}}\right)\right] \\
& =\frac{1}{4} \int_{0}^{\infty} \frac{d t}{\sqrt{t}} \int_{y_{0}}^{y_{M}} \frac{d y}{y}\left[\frac{1}{2} \Phi(t)+t \Phi^{\prime}(t)+\left(1-\chi_{S}\right) \Psi(t)\right] \\
& =\left(\ln y_{M}-\ln a\right)\left(1-\chi_{S}\right) g(0),
\end{aligned}
$$

where we have explicitly inserted $y_{0}$, and the terms with $\Phi$ vanish by a partial integration. Note that this term must be sufficient for the regularization and indeed is. In the second part of the integral of the next term we integrate out the $\theta_{1} \theta_{2}$ quantities, perform a partial integration, and get the result ( $\operatorname{set} \tau=a^{2} / y y_{M}$ )

$$
\begin{aligned}
\frac{1}{4} & \int_{1}^{\infty} \frac{d \tau}{\tau} \int_{4(\tau-1)}^{\infty} \frac{d t}{\sqrt{t}}\left[\frac{1}{2} \Phi(t)+t \Phi^{\prime}(t)+\left(1-\chi_{S}\right) \Psi(t)\right] \\
& =\frac{1}{4} \int_{1}^{\infty} d(\ln \tau) \int_{4(\tau-1)}^{\infty} \frac{d t}{\sqrt{t}}\left[\frac{1}{2} \Phi(t)+t \Phi^{\prime}(t)+\left(1-\chi_{S}\right) \Psi(t)\right] \\
& =-\left(1-\chi_{S}\right) g(0) \ln 2+\frac{1}{4} \int_{0}^{\infty} \frac{d t}{\sqrt{t}} \ln (t+4)\left[\frac{1}{2} \Phi(t)+t \Phi^{\prime}(t)+\left(1-\chi_{S}\right) \Psi(t)\right],
\end{aligned}
$$

and this contribution is independent of $a$ and $y_{M}$, respectively. Furthermore I have used the differentiation rule

$$
\frac{d}{d a} \int_{\psi(a)}^{\phi(a)} f(x, a) d x=f[\phi(a), a] \frac{d \phi(a)}{d a}+f[\psi(a), a] \frac{d \psi(a)}{d a}+\int_{\psi(a)}^{\phi(a)} \frac{d f(x, a)}{d a} d x .
$$

Let us consider the various terms. Firstly we have

$$
\begin{aligned}
& \frac{1}{4} \int_{0}^{\infty} \frac{d t}{\sqrt{t}} \ln (t+4)\left[\frac{1}{2} \Phi(t)+t \Phi^{\prime}(t)\right] \\
& \quad=-\frac{1}{4} \int_{0}^{\infty} \frac{\sqrt{t} \Phi(t)}{t+4} d t=\frac{1}{4 \pi} \int_{0}^{\infty} \frac{Q_{1}(w)}{w+4} d w \int_{0}^{w} \frac{\sqrt{t} d t}{(t+4) \sqrt{w-t}} \\
& \quad=\frac{1}{2} \int_{0}^{\infty} \tanh \frac{u}{2} \tanh \frac{u}{4}[g(u)-g(-u)] d u .
\end{aligned}
$$


Here I have used the integral [28, p.287]

$$
\int_{0}^{u} x^{\nu-1}(x+\alpha)^{\lambda}(u-x)^{\mu-1} d x=\alpha^{\lambda} u^{\mu+\nu-1} B(\mu, \nu)_{2} F_{1}\left(-\lambda, \nu, \mu+\nu ;-\frac{u}{\alpha}\right)
$$

together with [26, p.101]

$$
(1-z)^{1 / 2}{ }_{2} F_{1}\left(a, a+\frac{1}{2} ; 2 a ; z\right)={ }_{2} F_{1}\left(a-\frac{1}{2}, a ; 2 a ; z\right)=\left(\frac{1}{2}+\frac{1}{2} \sqrt{1-z}\right)^{1-2 a} .
$$

Next we get

$$
\begin{aligned}
\frac{1}{4} & \int_{0}^{\infty} \frac{d t}{\sqrt{t}} \ln (t+4) \Psi(t) \\
& =-\frac{1}{4 \pi} \int_{0}^{\infty} d w Q_{2}^{\prime}(w) \int_{0}^{w} \frac{\ln (t+4) d t}{\sqrt{t(w-t)}} \\
& =\frac{g(0) \ln 2}{2}+\frac{1}{32} \int_{0}^{\infty} \frac{Q_{2}(w) d w}{\sqrt{1+w / 4}(1+\sqrt{1+w / 4})} \\
& =\frac{g(0) \ln 2}{2}+\frac{1}{4} \int_{0}^{\infty} \tanh \frac{u}{4}\left\{[g(u)+g(-u)]-\tanh \frac{u}{2}[g(u)-g(-u)]\right\} d u
\end{aligned}
$$

Collecting terms we obtain

$$
\begin{aligned}
\frac{1}{4} & \int_{1}^{\infty} \frac{d \tau}{\tau} \int_{4(\tau-1)}^{\infty} \frac{d t}{\sqrt{t}}\left[\frac{1}{2} \Phi(t)+t \Phi^{\prime}(t)+\left(1-\chi_{S}\right) \Psi(t)\right] \\
= & -\frac{1}{2}\left(1-\chi_{S}\right) g(0) \ln 2 \\
& +\frac{1-\chi_{S}}{4} \int_{0}^{\infty} \tanh \frac{u}{4}[g(u)+g(-u)] d u+\frac{1+\chi_{S}}{4} \int_{0}^{\infty} \tanh \frac{u}{4} \tanh \frac{u}{2}[g(u)-g(-u)] d u \\
= & -\frac{1}{2}\left(1-\chi_{S}\right) g(0) \ln 2 \\
& +\frac{1-\chi_{S}}{4}\left\{\int_{0}^{\infty}[g(u)+g(-u)] d u-\frac{2}{\pi} \int_{-\infty}^{\infty} h(p)[\beta(1+2 i p)+\beta(1-2 i p)] d p\right\} \\
& +\frac{1+\chi_{S}}{4}\left\{\int_{0}^{\infty}[g(u)-g(-u)] d u-\frac{1}{\pi} \int_{-\infty}^{\infty} h(p)\left[\beta\left(\frac{1}{2}+i p\right)-\beta\left(\frac{1}{2}-i p\right)\right] d p\right\} .
\end{aligned}
$$

Here use has been made of the integrals [28, p.304, p.356]

$$
\int_{0}^{\infty} \frac{e^{-\mu x}}{1+e^{-x}} d x=\beta(\mu), \quad \int_{0}^{\infty} \frac{e^{-\mu x}}{\cosh x} d x=\beta\left(\frac{\mu+1}{2}\right),
$$


and $\beta(x)$ is the $\beta$-function defined by $\beta(x)=\frac{1}{2}\left[\Psi\left(\frac{1+x}{2}\right)-\Psi\left(\frac{x}{2}\right)\right]$, with $\Psi(z)=\Gamma^{\prime}(z) / \Gamma(z)$ the logarithmic derivative of the $\Gamma$-function. To finish the discussion we have to consider

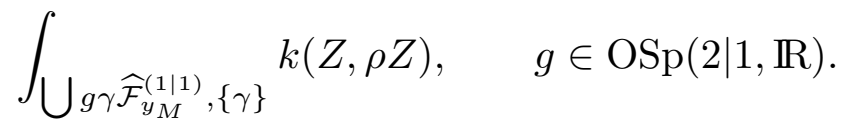

According to Venkov [71], this has the consequence that the asymptotic behaviour in the limit $y_{m} \rightarrow \infty$ is changed by a scaling such that the integral is calculated with respect to the variable $\nu y_{M}$ instead of $y_{M}$. The fixed number $\nu$ is denoted by $\nu(\rho)$. Similarly, $a$ is denoted by $a(\rho)$. Hence we obtain

$$
\begin{aligned}
& -\frac{1}{4} \int_{\widehat{\mathcal{F}}_{y_{M}}^{(1 \mid 1)}} d V(Z) \sum_{\begin{array}{c}
\{\rho\}, \widehat{\Gamma}_{\rho, e l l} \\
\operatorname{str}(\rho)+\chi_{\rho}=0
\end{array}} k\left(Z, \gamma^{\prime-1} \rho \gamma^{\prime} Z\right) \\
& =-\frac{1}{4} q(\widehat{\mathcal{F}})\left(1-\chi_{S}\right)\left(\ln y_{M}-\sum_{\substack{\{\rho\} ; \widehat{\Gamma}_{\rho, e l l} \\
\operatorname{str}(\rho)+\chi_{\rho}=0}} \ln \frac{a(\rho)}{\nu(\rho)}\right) g(0)+\frac{1}{8}\left(1-\chi_{S}\right) q(\widehat{\mathcal{F}}) g(0) \ln 2 \\
& \quad-\frac{1-\chi_{S}}{16} q(\widehat{\mathcal{F}}) \int_{0}^{\infty} \tanh \frac{u}{4}[g(u)+g(-u)] d u \\
& \quad-\frac{1+\chi_{S}}{16} q(\widehat{\mathcal{F}}) \int_{0}^{\infty} \tanh \frac{u}{4} \tanh \frac{u}{2}[g(u)-g(-u)] d u+o(1) .
\end{aligned}
$$

Here $q(\widehat{\mathcal{F}})$ denotes the number of classes $\{\rho\}$ having the property $\operatorname{str}(\rho)+\chi_{\rho}=0$ and $\rho$ elliptic. Because we know that all terms in the supertrace formula must be finite we deduce $q(\widehat{\mathcal{F}})=4 \kappa$. Therefore we obtain for the regularized "parabolic terms" in the Selberg supertrace formula for bordered super Riemann surfaces

$$
\begin{aligned}
\frac{1}{2} & \lim _{y_{m} \rightarrow \infty} \int_{\widehat{\mathcal{F}}_{y_{M}}^{(1 \mid 1)}} d V(Z)\left\{\sum_{\{S\}} \sum_{\gamma^{\prime} \in \widehat{\Gamma}_{S} \backslash \Gamma} k\left(Z, \gamma^{\prime-1} S \gamma^{\prime} Z\right)-\sum_{\substack{\{\rho\} ; \widehat{\Gamma}_{\rho, e l l} \\
\operatorname{str}(\rho)+\chi_{\rho}=0}} \sum_{\gamma^{\prime} \in \widehat{\Gamma}_{S} \backslash \Gamma} k\left(Z, \gamma^{\prime-1} \rho \gamma^{\prime} Z\right)\right\} \\
= & \kappa_{S} g(0)+\frac{\kappa_{-}}{2} \int_{0}^{\infty} g(-u) d u \\
& \left.-\frac{\kappa_{-}}{2} \int_{0}^{\infty} \ln \left(1-e^{-u}\right)\left\{\frac{d}{d u}[g(u)+g(-u))\right]\right\} d u+\frac{\kappa}{4} \int_{0}^{\infty}[g(u)-g(-u)] d u \\
& -\frac{\kappa_{-}}{4} \int_{0}^{\infty} \tanh \frac{u}{4}[g(u)+g(-u)] d u-\frac{\kappa_{+}}{4} \int_{0}^{\infty} \tanh \frac{u}{4} \tanh \frac{u}{2}[g(u)-g(-u)] d u .
\end{aligned}
$$

Here I have abbreviated

$$
\kappa_{S}=\frac{1}{2} \sum_{\{S\}}\left(1-\chi_{S}\right) \ln 2-\sum_{\substack{\{\rho\} ; \widehat{\Gamma}_{\rho, e l l} \\ \operatorname{str}(\rho)+\chi_{\rho}=0}} \ln \frac{a(\rho)}{\nu(\rho)} .
$$


Therefore we obtain

Theorem 4.2: The Selberg supertrace formula for the Dirac-Laplace operator $\square$ on bordered super Riemann surfaces with hyperbolic, elliptic and parabolic conjugacy classes with Dirichlet boundary-conditions is given by:

$$
\begin{aligned}
& \sum_{n=1}^{\infty}\left[h\left(p_{n}^{(B)}\right)-h\left(p_{n}^{(F)}\right)\right]=i \frac{\widehat{\mathcal{A}}}{4 \pi} \int_{-\infty}^{\infty} h(p) \tanh \pi p d p \\
& +\frac{1}{4} \sum_{\{\gamma\}} \sum_{k=1}^{\infty} \frac{l_{\gamma}}{\sinh \frac{k l_{\gamma}}{2}}\left[g\left(k l_{\gamma}\right)+g\left(-k l_{\gamma}\right)-\chi_{\gamma}^{k}\left(g\left(k l_{\gamma}\right) e^{-k l_{\gamma} / 2}+g\left(-k l_{\gamma}\right) e^{k l_{\gamma} / 2}\right)\right] \\
& -\frac{1}{4} \sum_{\left\{\rho^{2}\right\}} \sum_{k=0}^{\infty} \frac{l_{\rho^{2}}}{\cosh \left[\frac{1}{2}\left(k+\frac{1}{2}\right) l_{\rho^{2}}\right]}\left\{g\left[\left(k+\frac{1}{2}\right) l_{\rho^{2}}\right]+g\left[-\left(k+\frac{1}{2}\right) l_{\rho^{2}}\right]\right. \\
& \left.-\chi_{\rho^{2}}^{k+\frac{1}{2}}\left(g\left[\left(k+\frac{1}{2}\right) l_{\rho^{2}}\right] e^{-\frac{1}{2}\left(k+\frac{1}{2}\right) l} \rho_{\rho^{2}}+g\left[-\left(k+\frac{1}{2}\right) l_{\rho^{2}}\right] e^{\frac{1}{2}\left(k+\frac{1}{2}\right) l} \rho_{\rho^{2}}\right)\right\} \\
& -\frac{1}{2} \sum_{i=1}^{n} \sum_{k=1}^{\infty} \frac{l_{C_{i}}}{\cosh \frac{k l_{C_{i}}}{2}}\left\{g\left(k l_{C_{i}}\right)+g\left(-k l_{C_{i}}\right)-\chi_{C_{i}}^{k}\left(g\left(k l_{C_{i}}\right) e^{-k l_{C_{i}} / 2}+g\left(-k l_{C_{i}}\right) e^{k l_{C_{i}} / 2}\right)\right\} \\
& +\frac{1}{2} \sum_{\{R\}} \sum_{k=1}^{\nu-1} \frac{1}{\nu}\left\{\left(1-\chi_{R}^{k} \cos \frac{k \pi}{\nu}\right)\right. \\
& \left.\times \int_{0}^{\infty} \frac{g(u) e^{-u / 2}+g(-u) e^{u / 2}}{\cosh u-\cos (2 k \pi / \nu)} d u+\int_{0}^{\infty} \frac{g(u)-g(-u)}{\cosh u-\cos (2 k \pi / \nu)} \sinh \frac{u}{2} d u\right\} \\
& +\left(\kappa_{S}+\kappa_{-}\right) g(0)+\frac{\kappa_{-}}{2} \int_{0}^{\infty} g(-u) d u \\
& -\frac{\kappa_{-}}{2} \int_{0}^{\infty} \ln \left(1-e^{-u}\right)\left\{\frac{d}{d u}[g(u)+g(-u)]\right\} d u+\frac{\kappa}{4} \int_{0}^{\infty}[g(u)-g(-u)] d u \\
& -\frac{\kappa_{-}}{4} \int_{0}^{\infty} \tanh \frac{u}{4}[g(u)+g(-u)] d u-\frac{\kappa_{+}}{4} \int_{0}^{\infty} \tanh \frac{u}{4} \tanh \frac{u}{2}[g(u)-g(-u)] d u \text {, }
\end{aligned}
$$

where $\lambda_{n}^{(B, F)}=\frac{1}{2}+i p_{n}^{(B, F)}$ on the left runs through the set of all eigenvalues of this Dirichlet problem, and the summation on the right is taken over all primitive conjugacy classes $\{\gamma\}_{\widehat{\Gamma}}$, $\operatorname{str}(\gamma)+\chi_{\gamma}>2,\{R\}_{\widehat{\Gamma}}, \operatorname{str}(R)+\chi_{R}<2,\{\rho\}_{\widehat{\Gamma}}, \rho$ hyperbolic, $\{S\}_{\widehat{\Gamma}}, \operatorname{str}(S)+\chi_{S}=2$, and $\{\rho\}_{\widehat{\Gamma}}, \operatorname{str}(\rho)+\chi_{\rho}=0, \rho$ elliptic.

The test function $h$ is required to have the following properties

i) $h(p) \in C^{\infty}(\mathbb{R})$,

ii) $h(p)$ need not to be an even function in $p$,

iii) $h(p)$ vanishes faster than $1 /|p|$ for $p \rightarrow \pm \infty$.

iv) $h(p)$ is holomorphic in the strip $\Im(p) \leq \frac{1}{2}+\epsilon, \epsilon>0$, to guarantee absolute convergence in the summation over $\{\gamma\}$ and $\{\rho\}$. 
In the case of Neumann boundary-conditions the regularization procedure is similar to the treatment in Ref.[34] which is due to the fact that in this case the continuous spectrum does not drop out and must be taken into account. This will not discussed here again. The full picture emerges then by a proper combination of Ref.[34] and Theorem 4.2.

\section{Analytic Properties of Selberg Super Zeta-Functions}

The Selberg zeta-function was originally introduced by Selberg [63] in order to study spectra of Laplacians on compact Riemann surfaces of genus $g$. It is defined by

$$
Z(s):=\prod_{\{\gamma\}} \prod_{k=0}^{\infty}\left[1-e^{-(s+k) l_{\gamma}}\right], \quad(\Re(s)>1) .
$$

It is of further interest, because determinants of Laplacians can be expressed by combinations of the zeta-function and its derivatives. Define $D_{\Delta}(z)=\operatorname{det}^{\prime}(-\Delta+z)$, where the prime denotes the omission of zero modes. Then the Selberg zeta-function for compact closed Riemann surfaces and $D_{\Delta}$ are connected by the relation $[66,73]$ :

$$
Z(s)=s(s-1) D_{\Delta}[s(s-1)]\left[(2 \pi)^{1-s} e^{\widetilde{C}+s(s-1)} G(s) G(s+1)\right]^{2(g-1)},
$$

where $\widetilde{C}=\frac{1}{4}-\ln \sqrt{2 \pi}-2 \zeta^{\prime}(-1)$ and $G(z)$ denotes the Barnes $G$-function [28] (see also e.g. $[13,20,27])$. The case of non-compact closed and open Riemann surfaces can be found in Venkov [71] (also [12, 14]). We cite the latter case to have a comparison with its generalization to the super-case. The Selberg zeta-function for bordered Riemann surfaces for automorphic $m$-forms is defined by

$$
\begin{aligned}
& \hat{Z}(s)=\prod_{\{\gamma\}} \prod_{k=0}^{\infty}\left[1-\chi_{\gamma}^{m} e^{-l_{\gamma}(s+k)}\right] \\
& \times \prod_{\substack{\{\rho\} ; \widehat{\Gamma}_{\rho, h y p} \\
\operatorname{tr}(\rho) \neq 0}} \prod_{k=0}^{\infty}\left(\frac{1+\chi_{\rho}^{m} e^{-l_{\rho}(s+k)}}{1-\chi_{\rho}^{m} e^{-l_{\rho}(s+k)}}\right)^{(-1)^{k} \chi_{\mathcal{I}}^{m}} \times \prod_{i=1}^{n} \prod_{k=0}^{\infty}\left(\frac{1}{1-\chi_{C_{i}}^{m} e^{-l_{C_{i}}(s+k)}}\right)^{2(-1)^{k}}
\end{aligned}
$$

$\Re(s)>1$. Choosing the test-function

$$
h(p, s, b)=\frac{1}{\left(s-\frac{1}{2}\right)^{2}+p^{2}}-\frac{1}{\left(b-\frac{1}{2}\right)^{2}+p^{2}}
$$

yields 
Theorem 5.1 [12, 71]: The Selberg trace formula for the Selberg zeta-function on bordered Riemann surfaces for automorphic $m$-forms is given by:

$$
\begin{aligned}
\frac{\hat{Z}^{\prime}(s)}{\hat{Z}(s)}= & \left(s-\frac{1}{2}\right) \frac{2 \widehat{\mathcal{A}}}{\pi}\left[\Psi\left(s+\frac{m}{2}\right)+\Psi\left(s-\frac{m}{2}\right)-\Psi\left(b+\frac{m}{2}\right)-\Psi\left(b-\frac{m}{2}\right)\right] \\
& -i \sum_{\{R\}} \sum_{k=1}^{\nu-1} \frac{1}{\nu \sin (k \pi / \nu)} \sum_{l=0}^{\infty}\left[\frac{e^{-2 i(k \pi / \nu)(l+1 / 2-m / 2)}}{s+l-m / 2}-\frac{e^{2 i(k \pi / \nu)(l+1 / 2+m / 2)}}{s+l+m / 2}\right] \\
& +4\left(s-\frac{1}{2}\right) \sum_{j}\left(\frac{1}{\left(s-\frac{1}{2}\right)^{2}+p_{j}^{2}}-\frac{1}{\left(b-\frac{1}{2}\right)^{2}+p_{j}^{2}}\right)+\text { const }_{1}+\text { const }_{2}\left(s-\frac{1}{2}\right) \\
& +2 \kappa_{0} \Psi(1-s)-4 \kappa_{0}\left(s-\frac{1}{2}\right) \sum_{k=0}^{\infty} \frac{1}{\left(s-\frac{1}{2}\right)^{2}-\left(k+\frac{1}{2}\right)^{2}} \\
& +\kappa_{0}\left[\Psi(s)-\Psi\left(s+\frac{m}{2}\right)-\Psi\left(s+\frac{m}{2}\right)\right],
\end{aligned}
$$

with some constants const $t_{1,2}$.

The zero- and pole-structure can be read off, see Refs.[12, 71]. The functional equation has the form

$$
\hat{Z}(1-s)=\hat{Z}(s) \hat{\Psi}_{Z}(s)
$$

with the function $\hat{\Psi}_{Z}(s)$ given by

$$
\begin{aligned}
\hat{\Psi}_{Z}(s)= & {\left[\frac{\Gamma(1-s)}{\Gamma(s)}\right]^{2 \kappa} \exp \left\{-4 \widehat{\mathcal{A}} \int_{0}^{s-\frac{1}{2}} t\left(\begin{array}{c}
\tan (\pi t) \\
\cot (\pi t)
\end{array}\right) d t+4 \hat{c}\left(s-\frac{1}{2}\right)\right.} \\
+ & i \sum_{\{R\}} \sum_{k=1}^{\nu-1} \frac{1}{\nu \sin (k \pi / \nu)} \int_{0}^{s-1 / 2} \sum_{l=0}^{\infty}\left[\frac{e^{-2 i(k \pi / \nu)(l+1 / 2-m / 2)}}{s+l-m / 2}+\frac{e^{-2 i(k \pi / \nu)(l+1 / 2-m / 2)}}{s-l+(m-3) / 2}\right. \\
& \left.\left.-\frac{e^{-2 i(k \pi / \nu)(l+1 / 2+m / 2)}}{s+l+(m-1) / 2}+\frac{e^{-2 i(k \pi / \nu)(l+1 / 2+m / 2)}}{s-l-(m-3) / 2}\right] d t\right\},
\end{aligned}
$$

and the $\tan (\pi t)$-, respectively the $\cot (\pi t)$-term, has to be taken whether $m$ is even or odd, and the constant $\hat{c}$ given by

$$
\hat{c}=\frac{1}{4}\left[\sum_{\substack{\{\rho\} ; \widehat{\Gamma}_{\rho, \text { ell }} \\ \operatorname{tr}(\rho)=0}} \chi_{\rho}^{m} \ln \left(\frac{a(\rho)}{\mu(\rho)}\right)-\kappa_{0} \ln 2-\frac{L}{2}\right] .
$$


Let us consider the two Selberg super zeta-functions $Z_{0}$ and $Z_{1}$, respectively, defined by $[5,33]$

$$
\begin{aligned}
& Z_{0}(s)=\prod_{\{\gamma\}} \prod_{k=0}^{\infty}\left[1-e^{-(s+k) l_{\gamma}}\right], \\
& Z_{1}(s)=\prod_{\{\gamma\}} \prod_{k=0}^{\infty}\left[1-\chi_{\gamma} e^{-(s+k) l_{\gamma}}\right]
\end{aligned}
$$

for $\Re(s)>1$; and furthermore the functions

$$
\begin{aligned}
& R_{0}(s)=\frac{Z_{0}(s)}{Z_{0}(s+1)}=\prod_{\{\gamma\}}\left(1-e^{-s l_{\gamma}}\right), \\
& R_{1}(s)=\frac{Z_{1}(s)}{Z_{1}(s+1)}=\prod_{\{\gamma\}}\left(1-\chi_{\gamma} e^{-s l_{\gamma}}\right),
\end{aligned}
$$

for $\Re(s)>1$; the analytic properties of the $Z_{0,1}$-functions can be derived from the $R_{0,1^{-}}$ functions. The analytic properties for these functions for closed super Riemann surfaces were discussed in I and II. However, in the case of bordered super Riemann surfaces we will consider the modified Selberg super zeta-functions on bordered super Riemann surfaces

$$
\begin{aligned}
\hat{Z}_{0}(s)= & \prod_{\{\gamma\}} \prod_{k=0}^{\infty}\left[1-e^{-(s+k) l_{\gamma}}\right] \\
& \times \prod_{\substack{\{\rho\} \\
\operatorname{str}(\rho)+\chi_{\rho} \neq 0}} \prod_{k=0}^{\infty}\left(\frac{1+e^{-(s+k) l_{\rho}}}{1-e^{-(s+k) l_{\rho}}}\right)^{(-1)^{k}} \times \prod_{i=1}^{n} \prod_{k=0}^{\infty}\left(\frac{1}{1-e^{-l_{C_{i}}(s+k)}}\right)^{2(-1)^{k}} \\
\hat{Z}_{1}(s)= & \prod_{\{\gamma\}} \prod_{k=0}^{\infty}\left[1-\chi_{\gamma} e^{-(s+k) l_{\gamma}}\right] \\
& \times \prod_{\substack{\{\rho\} \\
\operatorname{str}(\rho)+\chi_{\rho} \neq 0}} \prod_{k=0}^{\infty}\left(\frac{1+\chi_{\rho} e^{-(s+k) l_{\rho}}}{1-\chi_{\rho} e^{-(s+k) l_{\rho}}}\right)^{(-1)^{k}} \times \prod_{i=1}^{n} \prod_{k=0}^{\infty}\left(\frac{1}{1-\chi_{C_{i}} e^{-l_{C_{i}}(s+k)}}\right)^{2(-1)^{k}}
\end{aligned}
$$

for $\Re(s)>1$. For convenience we will consider the functions

$$
\hat{R}_{0}(s):=\frac{\hat{Z}_{0}(s)}{\hat{Z}_{0}(s+1)}=\prod_{\{\gamma\}}\left(1-e^{-s l_{\gamma}}\right)
$$




$$
\begin{aligned}
& \times \prod_{\substack{\{\rho\} \\
\operatorname{str}(\rho)+\chi_{\rho} \neq 0}} \prod_{k=0}^{\infty}\left(\frac{1+e^{-(s+k) l_{\rho}}}{1-e^{-(s+k) l_{\rho}}}\right)^{\alpha_{k}(-1)^{k}} \times \prod_{i=1}^{n} \prod_{k=0}^{\infty}\left(\frac{1}{1-e^{-l_{C_{i}}(s+k)}}\right)^{2 \alpha_{k}(-1)^{k}} \\
\hat{R}_{1}(s) & :=\frac{\hat{Z}_{1}(s)}{\hat{Z}_{1}(s+1)}=\prod_{\{\gamma\}}\left(1-\chi_{\gamma} e^{-s l_{\gamma}}\right) \\
& \times \prod_{\{\rho\}} \prod_{k=0}^{\infty}\left(\frac{1+\chi_{\rho} e^{-(s+k) l_{\rho}}}{1-\chi_{\rho} e^{-(s+k) l_{\rho}}}\right)^{\alpha_{k}(-1)^{k}} \times \prod_{i=1}^{n} \prod_{k=0}^{\infty}\left(\frac{1}{1-\chi_{C_{i}} e^{-l_{C_{i}}(s+k)}}\right)^{2 \alpha_{k}(-1)^{k}} \\
& \operatorname{str}(\rho)+\chi_{\rho} \neq 0
\end{aligned}
$$

$\alpha_{k}=1(m=0), \alpha_{k}=2(k \in \mathbb{N})$, and $\Re(s)>1$. As we shall see, only functional relations for the $\hat{R}_{0,1}$-functions can be derived, but not for the $\hat{Z}_{0,1}$-functions.

1. The Selberg super zeta-function $R_{1}$. We first discuss the function $\hat{R}_{1}(s)$. In order to do this we choose the test function $[33](\Re(s, a)>1)$

$$
h_{1}(p, s, a)=2 i p\left(\frac{1}{s^{2}+p^{2}}-\frac{1}{a^{2}+p^{2}}\right)
$$

with the Fourier transformed function $g_{1}(u)$ given by

$$
g_{1}(u, s, a)=\operatorname{sign}(u)\left(e^{-s|u|}-e^{-a|u|}\right)
$$

The regularization term is needed to match the requirements of a valid test function in the trace formula. The relevant integrals have been already calculated in Refs.[33, 34], such that we just take the results. The exceptions are the new terms in the supertrace formula corresponding to the involuted orbits and the last summand in Eq.(4.38). For the latter we obtain by using the integrals (4.33)

$$
\begin{aligned}
\int_{0}^{\infty} & \tanh \frac{u}{4} \tanh \frac{u}{2}\left[g_{1}(u, s, a)-g_{1}(-u, s, a)\right] d u \\
& =4 \int_{0}^{\infty}\left(1-\frac{1}{\cosh u}\right)\left(e^{-2 s u}-e^{-2 a u}\right) d u \\
& =2\left[\frac{1}{s}-\frac{1}{a}-\Psi\left(\frac{s}{2}+\frac{3}{4}\right)+\Psi\left(\frac{s}{2}+\frac{1}{4}\right)+\Psi\left(\frac{a}{2}+\frac{3}{4}\right)-\Psi\left(\frac{a}{2}+\frac{1}{4}\right)\right] .
\end{aligned}
$$

Let us consider the term corresponding to the $\{\rho\}$-conjugacy classes in Eq.(4.38). We get 
by inserting $g_{1}(u)$ on the one hand

$$
\begin{aligned}
& -\frac{1}{4} \sum_{\left\{\rho^{2}\right\}} \sum_{k=0}^{\infty} \frac{l_{\rho^{2}}}{\cosh \left[\frac{1}{2}\left(k+\frac{1}{2}\right) l_{\rho^{2}}\right]}\left\{g\left[\left(k+\frac{1}{2}\right) l_{\rho^{2}}\right]+g\left[-\left(k+\frac{1}{2}\right) l_{\rho^{2}}\right]\right. \\
& \left.-\chi_{\rho^{2}}^{k+\frac{1}{2}}\left(g\left[\left(k+\frac{1}{2}\right) l_{\rho^{2}}\right] e^{-\frac{1}{2}\left(k+\frac{1}{2}\right) l_{\rho^{2}}}+g\left[-\left(k+\frac{1}{2}\right) l_{\rho^{2}}\right] e^{\frac{1}{2}\left(k+\frac{1}{2}\right) l_{\rho^{2}}}\right)\right\} \\
& =-\frac{1}{2} \sum_{\left\{\rho^{2}\right\}} l_{\rho^{2}} \sum_{k=0}^{\infty} \chi_{\rho^{2}}^{k+\frac{1}{2}} \tanh \left[\frac{1}{2}\left(k+\frac{1}{2}\right) l_{\rho^{2}}\right]\left(e^{-s\left(k+\frac{1}{2}\right) l_{\rho^{2}}}-e^{-a\left(k+\frac{1}{2}\right) l_{\rho^{2}}}\right) .
\end{aligned}
$$

On the other we have by means of

$$
\ln \frac{1+x}{1-x}=2 \sum_{k=0}^{\infty} \frac{x^{2 k+1}}{2 k+1}
$$

for the logarithmic derivative of the inverse hyperbolic terms of the $\hat{R}_{1}$-function

$$
\begin{aligned}
\frac{d}{d s} & \ln \prod_{\{\rho\}} \prod_{m=0}^{\infty}\left(\frac{1+\chi_{\rho} e^{-(s+m) l_{\rho}}}{1-\chi_{\rho} e^{-(s+m) l_{\rho}}}\right)^{\alpha_{m}(-1)^{m}} \\
= & 2 \sum_{\{\rho\}} \sum_{k=0}^{\infty} \frac{\chi_{\rho}^{2 k+1}}{2 k+1}\left(\frac{d}{d s} e^{-s l_{\rho}(2 k+1)}\right) \sum_{m=0}^{\infty} \alpha_{m}(-1)^{m} e^{-m l_{\rho}(2 k+1)} \\
= & -\sum_{\left\{\rho^{2}\right\}} l_{\rho^{2}} \sum_{k=0}^{\infty} \chi_{\rho^{2}}^{k+\frac{1}{2}} e^{-s l_{\rho^{2}}\left(k+\frac{1}{2}\right)}\left[1+2 \sum_{m=1}^{\infty}(-1)^{m} e^{-m l_{\rho^{2}}\left(k+\frac{1}{2}\right)}\right] \\
= & -\sum_{\left\{\rho^{2}\right\}} l_{\rho^{2}} \sum_{k=0}^{\infty} \chi_{\rho^{2}}^{k+\frac{1}{2}} e^{-s l_{\rho^{2}}\left(k+\frac{1}{2}\right)} \tanh \left[\frac{1}{2} l_{\rho^{2}}\left(k+\frac{1}{2}\right)\right] .
\end{aligned}
$$

And similarly for the third term in $\hat{R}_{1}$. Therefore we obtain the Selberg supertrace formula for the test function $h_{1}(p, s, a)$ as follows

$$
\begin{aligned}
& \frac{\hat{R}_{1}^{\prime}(s)}{\hat{R}_{1}(s)}-\frac{\hat{R}_{1}^{\prime}(a)}{\hat{R}_{1}(a)} \\
& =4 \sum_{n=1}^{\infty}\left[\frac{\lambda_{n}^{(B)}-\frac{1}{2}}{s^{2}-\left(\lambda_{n}^{(B)}-\frac{1}{2}\right)^{2}}-\frac{\lambda_{n}^{(B)}-\frac{1}{2}}{a^{2}-\left(\lambda_{n}^{(B)}-\frac{1}{2}\right)^{2}}-\frac{\lambda_{n}^{(F)}-\frac{1}{2}}{s^{2}-\left(\lambda_{n}^{(F)}-\frac{1}{2}\right)^{2}}+\frac{\lambda_{n}^{(F)}-\frac{1}{2}}{a^{2}-\left(\lambda_{n}^{(F)}-\frac{1}{2}\right)^{2}}\right] \\
& \quad-2 \sum_{\{R\}} \sum_{k=1}^{\nu-1} \frac{\chi_{R}^{k}}{\nu} \sum_{l=0}^{\infty} \cos \left[(2 l+1) \frac{k \pi}{\nu}\right]\left(\frac{1}{s+l+\frac{1}{2}}-\frac{1}{a+l+\frac{1}{2}}\right) \\
& \quad-\frac{\widehat{\mathcal{A}}}{2 \pi}\left[\Psi\left(s+\frac{1}{2}\right)-\Psi\left(a+\frac{1}{2}\right)\right]+\kappa\left(\frac{1}{s}-\frac{1}{a}\right) \\
& \quad-\kappa_{+}\left[\Psi\left(\frac{s}{2}+\frac{3}{4}\right)-\Psi\left(\frac{s}{2}+\frac{1}{4}\right)-\Psi\left(\frac{a}{2}+\frac{3}{4}\right)+\Psi\left(\frac{a}{2}+\frac{1}{4}\right)\right] .
\end{aligned}
$$


Thus we read off

Theorem 5.2: The Selberg super zeta-function $\hat{R}_{1}(s)$ is a meromorphic function on $\Lambda_{\infty}$ and has furthermore the following properties:

A) The Selberg super zeta-function $\hat{R}_{1}(s)$ has "trivial" zeros at the following points and nowhere else

i) $s=-\frac{1}{2}-l,(l=0,1,2, \ldots)$ and the multiplicity of these zeros is given by

$$
\# N_{l}=\frac{\widehat{\mathcal{A}}}{2 \pi}-2 \sum_{\{R\}} \sum_{k=1}^{\nu-1} \frac{\chi_{R}^{k}}{\nu} \sum_{l=0}^{\infty} \cos \left[(2 l+1) \frac{k \pi}{\nu}\right] .
$$

Note that if $\# N_{l}<0$, we have poles instead of zeros.

ii) $s=0$ with the multiplicity given by $\# N_{0}=\kappa$.

iii) $s=-\frac{3}{2}-2 l,(l=0,1,2, \ldots)$, with the multiplicity given by $\# N_{l}=2 \kappa_{+}$.

B) The Selberg super zeta-function $\hat{R}_{1}(s)$ has "trivial" poles at the following points and nowhere else

i) $s=-\frac{1}{2}-2 l l=0,-1,-2, \ldots$ with the multiplicity given by $\# P_{l}=2 \kappa_{+}$.

C) The Selberg super zeta-function $\hat{R}_{1}(s)$ has "non-trivial" zeros and poles at the following points and nowhere else [33]

i) $s=i p_{n}^{(B, F)}$ : there are zeros (poles) with twice the multiplicity as the corresponding eigenvalue of $\square$.

ii) $s=-i p_{n}^{(B, F)}$ : reversed situation for poles and zeros.

iii) $s=\lambda_{n}^{(B, F)}-\frac{1}{2}$ there are zeros (poles), and

iv) $s=-\left(\lambda_{n}^{(B, F)}-\frac{1}{2}\right)$ there are poles with twice the multiplicity as the corresponding eigenvalue of $\square$, respectively. The last two cases describe so-called small eigenvalues of the operator $\square$. All these "nontrivial" eigenvalues are supernumbers $s \in \Lambda_{\infty}$.

Of course, Eq.(5.23) can be extended meromorphically to all $s \in \Lambda_{\infty}$.

By means of the relation (5.16) the analytic properties of the Selberg super zeta-functions $\hat{Z}_{1}$ can be derived, compare also Ref.[33].

The test functions $h_{1}(p, s, a)$ is symmetric by the interchange $s \rightarrow-s$. Therefore subtracting the trace formula for $h_{1}(p, s, a)$ and $h_{1}(p,-s, a)$ yields the functional equation for $\hat{R}_{1}$ in differential form

$$
\begin{aligned}
& \frac{d}{d s} \ln \left[\hat{R}_{1}(s) \hat{R}_{1}(-s)\right] \\
& =-\frac{\widehat{\mathcal{A}}}{2} \tan \pi s-\kappa_{+}\left[\Psi\left(\frac{s}{2}+\frac{3}{4}\right)-\Psi\left(\frac{3}{4}-\frac{s}{2}\right)-\Psi\left(\frac{1}{4}+\frac{s}{2}\right)+\Psi\left(\frac{1}{4}-\frac{s}{2}\right)\right] \\
& \quad+\frac{2 \kappa}{s}-2 \sum_{\{R\}} \sum_{k=1}^{\nu-1} \frac{\chi_{R}^{k}}{\nu} \sum_{l=0}^{\infty} \cos \left[(2 l+1) \frac{k \pi}{\nu}\right]\left(\frac{1}{s+l+\frac{1}{2}}+\frac{1}{s-\left(l+\frac{1}{2}\right)}\right)
\end{aligned}
$$


[note $\left.\Psi\left(\frac{1}{2}+s\right)=\Psi\left(\frac{1}{2}-s\right)+\pi \tan \pi s\right]$. The integrated functional equation therefore has the form

$$
\hat{R}_{1}(s) \hat{R}_{1}(-s)=\text { const. }(\cos \pi s)^{\widehat{\mathcal{A}} / 2 \pi} s^{2 \kappa}\left[\frac{\Gamma\left(\frac{1}{4}+\frac{s}{2}\right) \Gamma\left(\frac{1}{4}-\frac{s}{2}\right)}{\Gamma\left(\frac{3}{4}-\frac{s}{2}\right) \Gamma\left(\frac{3}{4}+\frac{s}{2}\right)}\right]^{2 \kappa+} \hat{\Psi}_{1}(s),
$$

with the function $\hat{\Psi}_{1}(s)$ given by

$$
\hat{\Psi}_{1}(s)=\exp \left\{-2 \sum_{\{R\}} \sum_{k=1}^{\nu-1} \frac{\chi_{R}^{k}}{\nu} \sum_{l=0}^{\infty} \cos \left[(2 l+1) \frac{k \pi}{\nu}\right] \ln \left|s^{2}-\left(l+\frac{1}{2}\right)^{2}\right|\right\} \text {. }
$$

We can check the consistence of the functional equation with respect to the analytical properties of the Selberg super-zeta function $\hat{R}_{1}$. In the case that there are no elliptical and parabolic terms the functional equation simplifies into

$$
\hat{R}_{1}(s) \hat{R}_{1}(-s)=A_{1}(\cos \pi s)^{\widehat{\mathcal{A}} / 2 \pi}
$$

where $A_{1}$ is a constant given e.g. by $A_{1}=R_{1}\left(s_{0}\right) R_{1}\left(-s_{0}\right)\left(\cos \pi s_{0}\right)^{-\widehat{\mathcal{A}} / 2 \pi}$ with some $s_{0} \in \mathbb{C}$, which is however, independent of $s_{0}$.

2. The Selberg super zeta-function $\hat{R}_{0}$. Let us turn to the discussion of the Selberg super zeta-function $\hat{R}_{0}$. We consider the test-function $(\Re(s, a)>1)$

$$
h_{0}(p, s, a)=2\left(\frac{1}{2}+i p\right)\left(\frac{1}{s^{2}-\left(\frac{1}{2}+i p\right)^{2}}-\frac{1}{a^{2}-\left(\frac{1}{2}+i p\right)^{2}}\right),
$$

with the Fourier transform $g_{0}(u, s, a)$ given by

$$
g_{0}(u, s, a)=\operatorname{sign}(u) e^{u / 2}\left(e^{-s|u|}-e^{-a|u|}\right)
$$

Again the regularization term is needed to match the requirements of a valid test function for the trace formula. Similarly as in the previous case we obtain the Selberg super trace formula for the test function $h_{0}(p, s, a)$ as follows

$$
\begin{aligned}
& \frac{\hat{R}_{0}^{\prime}(s)}{\hat{R}_{0}(s)}-\frac{\hat{R}_{0}^{\prime}(a)}{\hat{R}_{0}(a)} \\
& =4 \sum_{n=1}^{\infty}\left[\frac{\lambda_{n}^{(B)}}{s^{2}-\left(\lambda_{n}^{(B)}\right)^{2}}-\frac{\lambda_{n}^{(B)}}{a^{2}-\left(\lambda_{n}^{(B)}\right)^{2}}-\frac{\lambda_{n}^{(F)}}{s^{2}-\left(\lambda_{n}^{(F)}\right)^{2}}+\frac{\lambda_{n}^{(F)}}{a^{2}-\left(\lambda_{n}^{(F)}\right)^{2}}\right] \\
& \quad-\sum_{\{R\}} \sum_{k=1}^{\nu-1} \frac{1}{\nu \sin (2 k \pi / \nu)} \sum_{l=1}^{\infty} \sin \left(\frac{2 l k \pi}{\nu}\right)\left[\frac{1}{s+l-1}-\frac{1}{s+l+1}-\frac{1}{a+l-1}+\frac{1}{a+l+1}\right] \\
& \quad-\frac{\widehat{\mathcal{A}}}{4 \pi}[\Psi(s)+\Psi(s+1)-\Psi(a)-\Psi(a+1)]
\end{aligned}
$$




$$
+\frac{\kappa}{2}\left(\frac{1}{s-\frac{1}{2}}+\frac{1}{s+\frac{1}{2}}-\frac{1}{a-\frac{1}{2}}-\frac{1}{a+\frac{1}{2}}-\frac{4}{s}+\frac{4}{a}\right)
$$

Note that no terms proportional to $\chi$ are present. Therefore we have shown

Theorem 5.3: The Selberg super zeta-function $\hat{R}_{0}(s)$ is a meromorphic function on $\Lambda_{\infty}$ and has furthermore the following properties:

A) The Selberg super zeta-function $\hat{R}_{0}(s)$ has "trivial" zeros at the following points and nowhere else: First note that

$$
\frac{1}{\sin (2 k \pi / \nu)} \sum_{l=1}^{\infty} \sin \left(\frac{2 l k \pi}{\nu}\right)\left(\frac{1}{s+l-1}-\frac{1}{s+l+1}\right)=\frac{1}{s}+\frac{\cos \left(\frac{2 k \pi}{\nu}\right)}{s+1}+2 \sum_{l=2}^{\infty} \frac{\cos \left(\frac{2 l k \pi}{\nu}\right)}{s+l}
$$

i) $s=0$ with multiplicity

$$
\# N_{0}=\frac{\widehat{\mathcal{A}}}{4 \pi}-2 \kappa-\sum_{\{R\}} \frac{\nu-1}{\nu}
$$

$s=-1$ with multiplicity

$$
\# N_{1}=\frac{\widehat{\mathcal{A}}}{2 \pi}-\sum_{\{R\}} \sum_{k=1}^{\nu-1} \frac{1}{\nu} \cos \left(\frac{2 k \pi}{\nu}\right)
$$

$s=-n(n=2,3, \ldots)$ with multiplicity

$$
\# N_{n}=\frac{\widehat{\mathcal{A}}}{2 \pi}-2 \sum_{\{R\}} \sum_{k=2}^{\nu-1} \frac{1}{\nu} \sum_{l=2}^{\infty} \cos \left(\frac{2 l k \pi}{\nu}\right) .
$$

Note that if $\# N_{n}<0$, we have poles instead of zeros.

ii) $s=-\frac{1}{2}$ with multiplicity $\# N_{-1 / 2}=\kappa / 2$.

iii) $s=\frac{1}{2}$ with multiplicity $\# N_{\frac{1}{2}}=\kappa / 2$.

B) The Selberg super zeta-function $\hat{R}_{0}(s)$ has "non-trivial" zeros and poles at the following points and nowhere else [33]

i) $s=i p_{n}^{(B, F)}+\frac{1}{2}$ : there are zeros (poles) with twice the multiplicity as the corresponding eigenvalue of $\square$.

ii) $s=-i p_{n}^{(B, F)}-\frac{1}{2}$ : reversed situation for poles and zeros.

iii) $s=\lambda_{n}^{(B, F)}$ there are zeros (poles), and

iv) $s=-\lambda_{n}^{(B, F)}$ there are poles with twice the multiplicity as the corresponding eigenvalue of $\square$, respectively. The last two cases describe so-called small eigenvalues of the operator $\square$. All these "nontrivial" eigenvalues are supernumbers $s \in \Lambda_{\infty}$.

Of course, Eq.(5.31) can be extended meromorphically to all $s \in \Lambda_{\infty}$. 
By means of the relation (5.15) the analytic properties of the Selberg super zeta-functions $\hat{Z}_{0}$ can be derived, compare also Ref.[33].

The test function $h_{0}(p, s, a)$ is symmetric with respect to $s \rightarrow-s$. Therefore subtracting the trace formulæ of $h_{0}(p, s, a)$ and $h_{0}(p,-s, a)$ from each other yields the functional equation for the $\hat{R}_{0}$-function in differential form

$$
\begin{aligned}
& \frac{d}{d s} \ln \left[\hat{R}_{0}(s) \hat{R}_{0}(-s)\right] \\
& =\frac{\widehat{\mathcal{A}}}{2 \pi} \frac{d}{d s} \ln (\sin \pi s)+\kappa\left(\frac{1}{s+\frac{1}{2}}+\frac{1}{s-\frac{1}{2}}-\frac{4}{s}\right) \\
& \quad-\sum_{\{R\}} \sum_{k=1}^{\nu-1} \frac{1}{\nu \sin (2 k \pi / \nu)} \sum_{l=1}^{\infty} \sin \left(\frac{2 l k \pi}{\nu}\right) \\
& \times\left[\frac{1}{s+l-1}+\frac{1}{s-(l-1)}-\frac{1}{s+l+1}-\frac{1}{s-(l+1)}\right] .
\end{aligned}
$$

In integrated form, this gives the functional equation

$$
\hat{R}_{0}(s) \hat{R}_{0}(-s)=\text { const. }(\sin \pi s)^{\widehat{\mathcal{A}} / 2 \pi}\left(\frac{s^{2}-\frac{1}{4}}{s^{4}}\right)^{\kappa} \hat{\Psi}_{0}(s),
$$

with the function $\hat{\Psi}_{0}(s)$ given by

$$
\hat{\Psi}_{0}(s)=\exp \left\{-\sum_{\{R\}} \sum_{k=1}^{\nu-1} \frac{1}{\nu \sin (2 k \pi / \nu)} \sum_{l=1}^{\infty} \sin \left(\frac{2 l k \pi}{\nu}\right) \ln \left|\frac{\left(s^{2}-(l-1)^{2}\right)}{\left(s^{2}-(l+1)^{2}\right)}\right|\right\} .
$$

We can check the consistence of the functional equation with respect to the analytical properties of the Selberg super-zeta function $\hat{R}_{0}$. In the case that there are no elliptical and parabolic terms the functional equation simplifies into

$$
\frac{\hat{Z}_{0}(s) \hat{Z}_{0}(-s)}{\hat{Z}_{0}(1+s) \hat{Z}_{0}(1-s)}=\hat{R}_{0}(s) \hat{R}_{0}(-s)=A_{0}(\sin \pi s)^{\widehat{\mathcal{A}} / 2 \pi}
$$

where the constant $A_{0}$ is e.g. given by $A_{0}=\hat{R}_{0}\left(s_{0}\right) \hat{R}_{0}\left(-s_{0}\right)\left(\sin \pi s_{0}\right)^{-\widehat{\mathcal{A}} / 2 \pi}$ with some $s_{0} \in$ $\mathbb{C}$, where $\widetilde{B}_{0}$ is independent of $s_{0}$.

3. The Selberg super zeta-function $\hat{Z}_{S}$. Following Refs.[33, 34, 47] we can also introduce the Selberg super zeta-function $\hat{Z}_{S}(s)$ defined by

$$
\hat{Z}_{S}(s)=\frac{\hat{Z}_{0}(s) \hat{Z}_{0}(s+1)}{\hat{Z}_{1}^{2}\left(s+\frac{1}{2}\right)}
$$

The appropriate test function is $(\Re(s)>1)$

$$
h_{S}(p, s)=\left.\frac{1}{s^{2}-\lambda^{2}}\right|_{\lambda=\frac{1}{2}+i p}=\frac{1}{\left(s^{2}-\frac{1}{4}\right)-i p+p^{2}}
$$


The corresponding Fourier transform $g_{S}$ is given by

$$
g_{S}(u, s)=\frac{1}{2 s} e^{u / 2-s|u|}
$$

The evaluation of the various terms in the Selberg supertrace formula is straightforward similarly to the previous two cases. We just present the evaluation of the fourth last term in Eq.(4.38) proportional to $\kappa_{-}$. We obtain

$$
\begin{aligned}
\int_{0}^{\infty} & \left.\ln \left(1-e^{-u}\right)\left\{\frac{d}{d u}[g(u)+g(-u))\right]\right\} d u \\
& =-\frac{1}{2 s} \int_{0}^{\infty} \ln \left(1-e^{-u}\right)\left[\left(s-\frac{1}{2}\right) e^{-u\left(s-\frac{1}{2}\right)}+\left(s+\frac{1}{2}\right) e^{-u\left(s+\frac{1}{2}\right)}\right] \\
& =-\frac{1}{2 s} \int_{0}^{1} \ln x\left[\left(s+\frac{1}{2}\right)(1-x)^{s-\frac{1}{2}}+\left(s-\frac{1}{2}\right)(1-x)^{s-\frac{3}{2}}\right] \\
& =\frac{1}{2 s}\left[2 C+\Psi\left(s+\frac{3}{2}\right)+\Psi\left(s+\frac{1}{2}\right)\right] .
\end{aligned}
$$

Here use has been made of the integral [28, p.538]

$$
\int_{0}^{1} x^{\mu-1}\left(1-x^{r}\right)^{\nu-1} \ln x d x=\frac{1}{r^{2}} B\left(\frac{\mu}{r}, \nu\right)\left[\Psi\left(\frac{\mu}{r}\right)-\Psi\left(\frac{\mu}{r}+\nu\right)\right] .
$$

Therefore we obtain

$$
\begin{aligned}
& \frac{1}{2 s} \frac{\hat{Z}_{S}^{\prime}(s)}{\hat{Z}_{S}(s)}=\frac{1}{2 s} \frac{d}{d s} \ln \left[\frac{\hat{Z}_{0}(s) \hat{Z}_{0}(s+1)}{\hat{Z}_{1}^{2}\left(s+\frac{1}{2}\right)}\right] \\
& =2 \sum_{n=1}^{\infty}\left[\frac{1}{s^{2}-\left(\lambda_{n}^{(B)}\right)^{2}}-\frac{1}{s^{2}-\left(\lambda_{n}^{(F)}\right)^{2}}\right]+\frac{\widehat{\mathcal{A}}}{8 \pi} \frac{1}{s^{2}} \\
& \quad-\frac{1}{2 s} \sum_{\{R\}} \sum_{k=1}^{\nu-1} \sum_{l=1}^{\infty} \frac{\sin (2 l k \pi / \nu)}{\nu \sin (2 k \pi / \nu)}\left[\frac{4\left(1-\chi_{R}^{k} \cos \left(\frac{k \pi}{\nu}\right)\right)}{s+l}+\frac{1}{s+l-1}+\frac{1}{s+l+1}-\frac{2}{s+l}\right] \\
& \quad+\frac{C \kappa_{-}-\kappa_{s}-\kappa_{-}}{s}+\frac{\kappa}{4 s}\left(\frac{4}{s}+\frac{1}{s-\frac{1}{2}}-\frac{1}{s+\frac{1}{2}}\right) \\
& \quad+\frac{\kappa_{-}}{s} \Psi(s)+\frac{\kappa_{+}}{2 s}\left[\Psi\left(\frac{s}{2}\right)-\Psi\left(\frac{s+1}{2}\right)\right] .
\end{aligned}
$$

This gives

Theorem 5.4: The Selberg super zeta-function $\hat{Z}_{S}$ is a meromorphic function on $\Lambda_{\infty}$ and has furthermore the following properties:

A) The Selberg super zeta-function $\hat{Z}_{S}(s)$ has "trivial" zeros at the following points and nowhere else 
i) $s=0$ with multiplicity

$$
\# N_{0}=\frac{\widehat{\mathcal{A}}}{4 \pi}-\sum_{\{R\}} \frac{\nu-1}{\nu}+2 \kappa
$$

$s=-1$ with multiplicity

$$
\# N_{1}=-2 \sum_{\{R\}} \sum_{k=1}^{\nu-1} \frac{1}{\nu}\left[1-2 \chi_{R} \cos \left(\frac{k \pi}{\nu}\right)+\cos \left(\frac{k \pi}{\nu}\right)\right]-4 \kappa .
$$

$s=-n(n=-2,3,4, \ldots)$ with multiplicity

$$
\# N_{n}=4 \sum_{\{R\}} \sum_{k=2}^{\nu-1} \frac{1}{\nu \sin (2 k \pi / \nu)}\left[\sin ^{2}\left(\frac{k \pi}{\nu}\right)-\left(1-\chi_{R} \cos \frac{k \pi}{\nu}\right)\right] \sin \left(\frac{2 l k \pi}{\nu}\right)-4 \kappa .
$$

ii) $s=\frac{1}{2}$ with multiplicity $\# N_{1 / 2}=\kappa / 2$.

iii) $s=-1-2 l, l=0,1,2, \ldots$, with multiplicity $\# N_{l}=2 \kappa_{+}$.

Note that if $\# N_{l}<0$, we have poles instead of zeros.

B) The Selberg super zeta-function $\hat{Z}_{S}(s)$ has "trivial" poles at the following points and nowhere else

i) $s=-\frac{1}{2}$ with multiplicity $\# P_{-1 / 2}=\kappa / 2$.

C) The Selberg super zeta-function $\hat{Z}_{S}(s)$ has "non-trivial" zeros and poles at the following points and nowhere else [33, 47]

i) $s= \pm\left(\frac{1}{2}+i p_{n}^{(B)}\right)$ there are zeros and

ii) $s= \pm\left(\frac{1}{2}+i p_{n}^{(F)}\right)$ there are poles, with twice the multiplicity as the corresponding eigenvalue of $\square$, respectively.

Of course, Eq.(5.44) can be extended meromorphically to all $s \in \Lambda_{\infty}$.

The test function $h_{S}(p, s)$ is symmetric with respect to $s \rightarrow-s$ and therefore we can deduce the functional relation

$$
\begin{aligned}
& \frac{\hat{Z}_{S}(s)}{\hat{Z}_{S}(-s)}=\text { const. } e^{4 s\left(C \kappa_{-}-\kappa_{S}-\kappa_{-}\right)} \\
& \quad \times\left(\frac{s-\frac{1}{2}}{s+\frac{1}{2}}\right)^{\kappa}\left(\frac{\Gamma(s)}{\Gamma(-s)}\right)^{2 \kappa-}\left(\frac{\Gamma\left(\frac{s}{2}\right) \Gamma\left(\frac{1}{2}-\frac{s}{2}\right)}{\Gamma\left(\frac{1}{2}-\frac{s}{2}\right) \Gamma\left(-\frac{s}{2}\right)}\right)^{2 \kappa_{+}} \hat{\Psi}_{S}(s),
\end{aligned}
$$

with the function $\hat{\Psi}_{S}(s)$ given by

$$
\begin{aligned}
\hat{\Psi}_{S}(s)=\exp & \left\{-2 \sum_{\{R\}} \sum_{k=1}^{\nu-1} \frac{1}{\nu \sin (2 k \pi / \nu)} \sum_{l=1}^{\infty} \sin \left(\frac{2 l k \pi}{\nu}\right)\right. \\
& \left.\times\left[2\left(1-2 \chi_{R}^{k} \cos \frac{k \pi}{\nu}\right) \ln \left|\frac{s+l}{s-l}\right|+\ln \left|\frac{(s+l-1)(s+l+1)}{(s-l+1)(s-l-1)}\right|\right]\right\} .
\end{aligned}
$$


We can check the consistence of the functional equation with respect to the analytical properties of the Selberg super-zeta function $\hat{Z}_{S}$. In the case, where only hyperbolic conjugacy classes are present in the super Fuchsian group, Eq.(5.48) reduces to the simple functional equation [33]

$$
\hat{Z}_{S}(s)=\hat{Z}_{S}(-s) .
$$

Let us note that the relation

$$
\begin{aligned}
& \frac{d}{d s} \ln \left[\frac{\hat{Z}_{0}(s) \hat{Z}_{0}(s+1)}{\hat{Z}_{1}\left(s+\frac{1}{2}\right)}\right]-\frac{d}{d s} \ln \left[\frac{\hat{Z}_{0}(s+1) \hat{Z}_{0}(s+2)}{\hat{Z}_{1}\left(s+\frac{3}{2}\right)}\right] \\
&=\frac{\hat{R}_{0}^{\prime}(s)}{\hat{R}_{0}(s)}+\frac{\hat{R}_{0}^{\prime}(s+1)}{\hat{R}_{0}(s+1)}-2 \frac{\hat{R}_{1}^{\prime}\left(s+\frac{1}{2}\right)}{\hat{R}_{1}\left(s+\frac{1}{2}\right)}
\end{aligned}
$$

provides a consistency check for the zeta functions $\hat{R}_{0}, \hat{R}_{1}$ and $\hat{Z}_{S}$, respectively.

Let us note that in the case of Neumann boundary-conditions the Selberg super zetafunctions must be differently defined due to the changed signs of the $\gamma \mathcal{I}$-terms, i.e. the power of the corresponding terms in the zeta-functions is reversed. This concludes the discussion.

\section{Super-Determinants of Dirac-Laplace Operators}

Since $\square_{m}^{2}$ is not a positive definite operator I calculate the super-determinants of $\left(c^{2}-\square_{m}^{2}\right)$ for $\Re(c)>0$ and analytically continue in $c$. Similar considerations have been done by Aoki [3] and Ref.[33] by means of the supertrace of the heat kernel of $\square_{m}^{2}$ for the case of closed super Riemann surfaces.

The super-determinant is defined using the $\zeta$-function regularization in the following way

$$
\begin{aligned}
\operatorname{sdet}\left(c^{2}-\square_{m}^{2}\right) & =\exp \left[-\left.\frac{\partial}{\partial s} \zeta(s ; c)\right|_{s=0}\right] \\
\zeta(s ; c) & =\operatorname{str}\left[\left(c^{2}-\square_{m}^{2}\right)^{-s}\right]=\frac{1}{\Gamma(s)} \int_{0}^{\infty} d t t^{s-1} \operatorname{str}\left\{\exp \left[-t\left(c^{2}-\square_{m}^{2}\right)\right]\right\}
\end{aligned}
$$

The function $h$ corresponding to the heat-kernel of $\left(c^{2}-\square_{m}^{2}\right)$ reads $h_{h k}(s)=e^{t\left[(s+m / 2)^{2}-c^{2}\right]}$. This gives

$$
G(u, \chi)=\frac{1}{\sqrt{\pi t}} e^{-u^{2} / 4 t-c^{2} t}\left[\cosh (m+1) \frac{u}{2}-\chi \cosh \frac{u m}{2}\right] .
$$

Splitting now the calculation of $\zeta(s ; c)$ into two terms corresponding to the identity transformation and the length term, respectively, gives:

$$
\zeta(s ; c)=\zeta_{I}(s ; c)+\zeta_{\Gamma}(s ; c) .
$$

We have $[3,33]$

$$
\zeta_{I}(s ; c)=-\frac{\widehat{\mathcal{A}}}{8 \pi \Gamma(s)} \sum_{k=0}^{m} \int_{0}^{\infty} t^{s-1} e^{-\left(c^{2}-k^{2}\right) t}=-\frac{\widehat{\mathcal{A}}}{8 \pi} \sum_{k=0}^{m}\left(c^{2}-k^{2}\right)^{-s},
$$


therefore

$$
\left.\frac{\partial}{\partial s} \zeta_{I}(s ; c)\right|_{s=0}=\frac{\widehat{\mathcal{A}}}{8 \pi} \sum_{k=0}^{m} \ln \left(c^{2}-k^{2}\right) .
$$

Let us consider the representation $(\Re(s)<1)$ :

$$
t^{s-1}=\frac{2}{\Gamma(1-s)} \int_{0}^{\infty} \frac{\lambda+c}{[\lambda(\lambda+2 c)]^{s}} e^{-\lambda(\lambda+2 c) t} d \lambda ;
$$

Therefore we get for the $\zeta_{\Gamma}(c ; s)$-contribution $[3,33]$ in Eq.(6.1) ( $m$ even)

$$
\zeta_{\Gamma}(s ; c)=\frac{\sin \pi s}{8 \pi} \int_{0}^{\infty} \frac{d \lambda}{[\lambda(\lambda+2 c)]^{s}} \frac{d}{d \lambda} \ln \left[\frac{\hat{Z}_{0}\left(\lambda+c+1+\frac{m}{2}\right) \hat{Z}_{0}\left(\lambda+c-\frac{m}{2}\right)}{\hat{Z}_{1}\left(\lambda+c+\frac{1-m}{2}\right) \hat{Z}_{1}\left(\lambda+c+\frac{m+1}{2}\right)}\right],
$$

where the logarithmic derivative of the super zeta-functions has been used. Let be $f(s)=$ $\left.\sin (\pi s)[\lambda(\lambda+2 c)]^{-s}\right]$. Then $\left.f^{\prime}(s)\right|_{s=0}=\pi$ and we get for $\zeta^{\prime}(0 ; c)(\Re(s)>0)$ :

$$
\zeta^{\prime}(0 ; c)=\frac{\widehat{\mathcal{A}}}{8 \pi} \ln c^{2}-\frac{1}{2} \ln \left[\frac{\hat{Z}_{0}\left(c+1+\frac{m}{2}\right) \hat{Z}_{0}\left(c-\frac{m}{2}\right)}{\hat{Z}_{1}\left(c+\frac{1-m}{2}\right) \hat{Z}_{1}\left(c+\frac{m+1}{2}\right)}\right] .
$$

Performing the limit $c \rightarrow \epsilon$ for $|\epsilon| \ll 1$, I get for $m=0$ and $m=-1$, respectively

$$
\begin{aligned}
\operatorname{sdet}\left(-\square_{0}^{2}\right) & =\pi^{\widehat{\mathcal{A}} / 4 \pi} \frac{\hat{Z}_{0}(1)}{\hat{Z}_{1}\left(\frac{1}{2}\right)} \sqrt{\frac{\hat{Z}_{1}(0)}{\hat{Z}_{1}(1)}}, \\
\operatorname{sdet}\left(-\square_{-1}^{2}\right) & =\pi^{-\widehat{\mathcal{A}} / 4 \pi} \frac{\hat{Z}_{1}\left(\frac{1}{2}\right)}{\hat{Z}_{0}(1)} \sqrt{\frac{\hat{Z}_{1}(1)}{\hat{Z}_{1}(0)}} .
\end{aligned}
$$

In the general case we obtain

$$
\begin{array}{rlr}
\operatorname{sdet}\left(-\square_{m}^{2}\right) & =\left(\frac{\pi}{m !}\right)^{\widehat{\mathcal{A}} / 4 \pi} \frac{\hat{Z}_{0}\left(1+\frac{m}{2}\right)}{\hat{Z}_{1}\left(\frac{m+1}{2}\right)} \sqrt{\frac{\hat{Z}_{1}(0)}{\hat{Z}_{1}(1)}}, & m=2,4, \ldots \\
\operatorname{sdet}\left(-\square_{-m}^{2}\right) & =\left(\frac{(m-2) !}{\pi}\right)^{\widehat{\mathcal{A}} / 4 \pi} \frac{\hat{Z}_{0}\left(\frac{m}{2}\right)}{\hat{Z}_{1}\left(\frac{m+1}{2}\right)} \sqrt{\frac{\hat{Z}_{1}(1)}{\hat{Z}_{1}(0)}}, & m=2,4, \ldots \\
\operatorname{sdet}\left(-\square_{m}^{2}\right) & =\left(\frac{\pi}{i m !}\right)^{\widehat{\mathcal{A}} / 4 \pi} \frac{\hat{Z}_{1}\left(1+\frac{m}{2}\right)}{\hat{Z}_{0}\left(\frac{m+1}{2}\right)} \sqrt{\frac{\hat{Z}_{1}(0)}{\hat{Z}_{1}(1)}}, & m=1,3, \ldots \\
\operatorname{sdet}\left(-\square_{-m}^{2}\right) & =\left(\frac{i(m-2) !}{\pi}\right)^{\widehat{\mathcal{A}} / 4 \pi} \frac{\hat{Z}_{1}\left(\frac{m}{2}\right)}{\hat{Z}_{0}\left(\frac{m+1}{2}\right)} \sqrt{\frac{\hat{Z}_{1}(1)}{\hat{Z}_{1}(0)}}, & m=3,5, \ldots
\end{array}
$$

(note for instance the relation $\operatorname{sdet}\left(-\square_{0}^{2}\right) \cdot \operatorname{sdet}\left(-\square_{-1}^{2}\right)=1$ ). Here, of course, use has been made of the functional relations for the modified Selberg super zeta-functions. In particular we get [c.f. Eq.(1.3)]

$$
\left[\operatorname{sdet}\left(-\square_{0}^{2}\right)\right]^{-5 / 2}\left[\operatorname{sdet}\left(-\square_{2}^{2}\right)\right]^{1 / 2}=\frac{\pi^{-\widehat{\mathcal{A}} / 2 \pi}}{\sqrt{2}}\left(\frac{\hat{Z}_{0}(1)}{\hat{Z}_{1}\left(\frac{1}{2}\right)}\right)^{-5 / 2}\left(\frac{\hat{Z}_{0}(2)}{\hat{Z}_{1}\left(\frac{3}{2}\right)}\right)^{1 / 2} \frac{\hat{Z}_{1}(0)}{\hat{Z}_{1}(1)},
$$


These determinants are the ones for the Dirac-Laplace operator for Dirichlet boundaryconditions on bordered super Riemann surface. In order to distinguish from the those with Neumann boundary-conditions, $\operatorname{sdet}_{\Sigma}^{(N) \prime}\left(-\square_{0}^{2}\right)$, we denote therefore $\operatorname{sdet}\left(-\square_{0}^{2}\right) \equiv$ $\operatorname{sdet}_{\Sigma}^{(D)}\left(-\square_{0}^{2}\right)$. Now we know that the Selberg super zeta-functions have concerning the $\gamma \mathcal{I}$-length product the reverse power behaviour, denoted by an index " $(N)$ ", i.e. $Z^{(N)}(s)$. Furthermore we have to take into account that instead of bosonic and fermionic eigenfunctions which are odd with respect to $x$ of $\square_{0}$, we have that bosonic and fermionic eigenfunctions which are even with respect to $x$ appear, i.e. we have for instance

$$
\operatorname{sdet}_{\Sigma}^{(N) \prime}\left(-\square_{0}^{2}\right)=(-1)^{1-2 q} \pi^{\widehat{\mathcal{A}} / 4 \pi} \frac{\hat{Z}_{0}^{(N)}(1)}{\widetilde{Z}_{1}^{(N)}\left(\frac{1}{2}\right)} \sqrt{\frac{\hat{Z}_{0}^{(N)}(0)}{\hat{Z}_{0}^{(N)}(1)}}
$$

Here by $\widetilde{Z}_{1}^{(N)}\left(\frac{1}{2}\right)$ the order of $\hat{Z}_{1}^{(N)}$ at $s=\frac{1}{2}$ is denoted, depending whether $\Delta n_{0}^{(0)} \leq 0$ or $\Delta n_{0}^{(0)}>0$, respectively. $\Delta n_{0}^{(0)}=n_{0}^{B}-n_{0}^{F}$ denotes the difference between the number of even bosonic- and fermionic zero-modes of the Dirac-Laplace operator $\square_{0}$. According to Ref.[52] $\Delta n_{0}^{(0)}=1-2 q$ with $q=\operatorname{dim}$ ker $\bar{\partial}_{1}$ and $\bar{\partial}_{p}^{\dagger}=-y^{2} \partial_{z}+\frac{1}{2} p y$. From the corresponding expressions for $\operatorname{sdet}_{\Sigma}^{(D)}\left(-\square_{m}^{2}\right)$ and $\operatorname{sdet}_{\Sigma}^{(N){ }^{\prime}}\left(-\square_{m}^{2}\right)$, respectively, now follows

$$
\operatorname{sdet}_{\Sigma}^{(D)}\left(-\square_{m}^{2}\right) \cdot \operatorname{sdet}_{\Sigma}^{(N) \prime}\left(-\square_{m}^{2}\right)=\operatorname{sdet}_{\widehat{\Sigma}}{ }^{\prime}\left(-\square_{m}^{2}\right),
$$

where $\widehat{\Sigma}$ denotes the closed double of the bordered super Riemann surface. The corresponding Selberg super zeta-functions on $\widehat{\Sigma}$ are then defined by [33]

$$
Z_{q}(s)=\prod_{\{\gamma\}} \prod_{k=0}^{\infty}\left(1-\chi_{\gamma}^{q} e^{-(s+k) l_{\gamma}}\right)
$$

$(q=0,1)$. Equation (6.17) shows in a nice way the super-analogue of Eq.(1.2) and concludes the discussion.

\section{Discussion and Summary}

In this paper I have discussed a super extension of the Selberg trace formula for bordered Riemann surfaces, the Selberg supertrace formula for bordered super Riemann surfaces, including hyperbolic, elliptic and parabolic conjugacy classes. In the case of the incorporation of parabolic conjugacy classes an appropriate regularization scheme had to be applied. In the case of Dirichlet boundary-conditions, Eisenstein series representing the continuous spectrum of the Dirac-Laplace operator $\square$ were not needed because the have even parity with respect to $x$ and they dropped out.

Furthermore, I could discuss Selberg super zeta-functions. Similarly as in the usual case, there appeared additional "trivial zeros" and "trivial poles" in comparison to the "trivial zeros" of the super zeta-function due to the additional elliptic and parabolic conjugacy classes. In particular, the elliptic and parabolic conjugacy classes altered the multiplicity of the trivial poles already due to the hyperbolic conjugacy classes, the parabolic terms introduced new structure. 
I also could calculate super-determinants of the Dirac-Laplace operators on bordered super Riemann surfaces, for Dirichlet and Neumann boundary-conditions respectively. These determinants were expressed in terms of the Selberg super-zeta functions which gave a closed expression for the integrand in the Polyakov path integral for open fermionic strings. Within this formalism it could also be shown that the product of these determinants gave the determinants of the Dirac-Laplace operators on the doubled surface.

However, there are still open problems and questions. For instance, it is not difficult to derive the corresponding so-called Weyl's and Huber's laws for the increase in the number of the energy levels and the norms of the hyperbolic conjugacy classes, respectively. Whereas in the former case one gets $\# N\left[\lambda_{n}^{(B)}-\lambda_{n}^{(F)}\right] \propto C A / 4 \pi(\lambda \rightarrow \infty)$, which gives a Wittenindex, in the latter one finds $\# N(L) \propto e^{\Delta n_{0}^{(0)} L} / L(L \rightarrow \infty)$ form which follows $\Delta n_{0}^{(0)}>0$.

The case of super automorphic $m$-forms $(m \neq 0)$ is only available for hyperbolic conjugacy classes, which are the most important ones due to their appearance in the evaluation of determinants. In order to set up the corresponding supertrace formula which include elliptic and parabolic conjugacy classes as well, the inversion formulæ Eqs. $(3.32,3.34)$ must be exploited which are very involved and give rise to considerably complicated expressions.

Finally, we must note that it is not clear for general non-cocompact (super) Fuchsian groups, whether the Maass-Laplacian $\Delta_{m}$ and its super counterpart $\square_{m}$ have infinitely many eigenvalues. In fact, for non-arithmetic groups a conjecture by Phillips and Sarnak [55] says that this will not be the case. In the case of arithmetic groups it is then possible to evaluate all contributions needed for the calculation of determinants in the trace formula [42]. However, it is nevertheless possible to define (super-) determinants by means of Selberg (super) zeta-functions which are constructed in the usual way (see Takhtajan and Zograf [67] for a discussion). However, a treatment of this matter for the case of (bordered) super Riemann surfaces is beyond the scope of this paper and is devoted to future investigations.

\section{Acknowledgement}

Fruitful discussions are gratefully acknowledged with J. Bolte, H. Ninnemann, C. Reina and F. Steiner. Special thanks are given to D. Hejhal, and I thank K. Aoki and S. B. Giddings for helpful communications. I would also like to thank the members of the II.Institut für Theoretische Physik, Hamburg University, for their kind hospitality.

\section{References}

[1] Alvarez, O.: Theory of Strings with Boundaries: Fluctuations, Topology and Quantum Theory. Nucl.Phys. B 216, 125-184 (1983)

[2] Alvarez-Gaumé, L., Moore, G. and Vafa, C.: Theta Functions, Modular Invariance, and Strings. Commun.Math.Phys. 106, 1-40 (1986)

[3] Aoki, K.: Heat Kernels and Super Determinants of Laplace Operators on Super Riemann Surfaces. Commun.Math.Phys. 117, 405-429 (1988)

[4] Baranov, A.M., Manin, Yu.I., Frolov, I.V. and Schwarz, A.S.: The Multiloop Contribution in the Fermionic String. Sov.J.Nucl.Phys. 43, 670-671 (1986)

[5] Baranov, A.M., Manin, Yu.I., Frolov, I.V. and Schwarz, A.S.: A Superanalog of the Selberg Trace Formula and Multiloop Contributions for Fermionic Strings. Commun.Math.Phys. 111, 373-392 (1987) 
[6] Baranov, A.M., Frolov, I.V. and Shvarts, A.S.: Geometry of Two-Dimensional Superconformal Field Theories. Theor.Math.Phys. 70, 64-72 (1987)

[7] Baranov, A.M. and A.S.Schwarz, A.S.: Multiloop Contribution to String Theory. JETP Lett. 42, 419-421 (1985); On the Multiloop Contributions to the String Theory. Int.J.Mod.Phys. A 2, 1773$1796(1987)$

[8] Batchelor, M.: The Structure of Supermanifolds. Trans.Amer.Math.Soc. 253, 329-338 (1979); Two Approaches to Supermanifolds. Trans.Amer.Math.Soc. 258, 257-270 (1980)

[9] Batchelor, M. and Bryant, P.: Graded Riemann Surfaces. Commun.Math.Phys. 114, 243-255 (1988)

[10] Blau, S.K. and Clements, M.: Determinants of Laplacians for World Sheets with Boundaries. Nucl. Phys. B 284, 118-130 (1987)

[11] Blau, S.K., Clements, M., Della Pietra, S., Carlip, S. and Della Pietra, V.: The String Amplitude on Surfaces with Boundaries and Crosscaps. Nucl.Phys. B 301, 285-303 (1988)

[12] Bolte, J. and Grosche, C.: Selberg Trace Formula for Bordered Riemann Surfaces: Hyperbolic, Elliptic and Parabolic Conjugacy Classes, and Determinants of Maass-Laplacians; DESY-Preprint DESY 92 - 118, and SISSA-Preprint, SISSA/139/92/FM, August 1992

[13] Bolte, J. and Steiner, F.: Determinants of Laplace-Like Operators on Riemann Surfaces. Commun. Math.Phys. 130, 581-597 (1990)

[14] Bolte, J. and Steiner, F.: The Selberg Trace Formula for Bordered Riemann Surfaces. DESY Preprint, DESY 90-082, 1-14, July 1990

[15] Burgess, C. and Morris, T.R.: Open and Unoriented Strings à la Polyakov. Nucl.Phys. B 291, 256-284 (1987); Open Superstrings à la Polyakov. Nucl.Phys. B 291, 285-333 (1987)

[16] S.Carlip: Sewing Closed String Amplitudes. Phys.Lett. B 209 464-472 (1988)

[17] De Beer, W. and Rodriguez, J.P.: Holomorphic Factorization and Open Strings. Phys.Lett. B 213, 291-297 (1988)

[18] DeWitt, B.: Supermanifolds. Cambridge: Cambridge University Press, 1984

[19] D'Hoker, E. and Phong, D.H.: Loop Amplitudes for the Bosonic Polyakov String. Nucl.Phys. B 269, 205-234 (1986)

[20] D'Hoker, E. and Phong, D.H.: On Determinants of Laplacians on Riemann Surfaces. Commun.Math. Phys. 104, 537-545 (1986)

[21] D'Hoker, E. and Phong, D.H.: Loop Amplitudes for the Fermionic String. Nucl.Phys. B 278, 225-241 (1986)

[22] D'Hoker, E. and Phong, D.H.: The Geometry of String Perturbation Theory. Rev.Mod.Phys. 60, 917-1065 (1988)

[23] Dunbar, D.C.: Boundary and Crosscap States in Compactification of Open and Closed Bosonic Strings. Int.J.Mod.Phys. A 4, 5149-5176 (1989)

[24] Efrat, I.: Determinants of Laplacians on Surfaces of Finite Volume. Commun.Math.Phys. 119, 443-451 (1988); and Erratum: Commun.Math.Phys. 119, 607 (1991)

[25] Elstrodt, J.: Die Resolvente zum Eigenwertproblem der automorphen Formen in der hyperbolischen Ebene. Teil I. Math.Ann. 203, 295-330 (1973)

[26] Erdélyi, A., Magnus, W., Oberhettinger, F., and Tricomi, F.G. (eds.): Higher Transcendental Functions, Vol.I. New York: McGraw Hill, 1954

[27] Gilbert, G.: String Theory Path Integral - Genus Two and Higher. Nucl.Phys. B 277, 102-124 (1986)

[28] Gradshteyn, I.S. and Ryzhik, I.M.: Table of Integrals, Series, and Products. New York: Academic Press, 1980

[29] Green, M.B.: Supersymmetrical Dual String Theories and Their Field Theory Limits. Surveys in High Energy Physics 3, 127-160 (1983)

[30] Green, M.B. and Schwarz, J.H.: Supersymmetrical Dual String Theory, I-III. Nucl. Phys. B 181, 502-530 (1982), B 198, 252-268, 441-460 (1982) 
[31] Green, M.B. and Schwarz, J.H.: Anomaly Cancelations in Supersymmetric D=10 Gauge Theory and Superstring Theory. Phys.Lett. B 149, 117-122 (1984); Infinity Cancelations in SO(32) Superstring Theory. Phys.Lett. B 151, 21-25 (1985); The Hexagon Gauge Anomaly in Type I Superstring Theory. Nucl.Phys. B 255, 93-114 (1985)

[32] Green, M.B., Schwarz, J.H. and Witten, E.: Superstring Theory I\&II. Cambridge: Cambridge University Press, 1988

[33] Grosche, C.: Selberg Supertrace Formula for Super Riemann Surfaces, Analytic Properties of the Selberg super zeta-functions and Multiloop Contributions to the Fermionic String. DESY Preprint DESY 89 - 010, February 1989, 1-102, and Commun.Math.Phys. 133, 433-485 (1990)

[34] Grosche, C.: Selberg Supertrace Formula for Super Riemann Surfaces II: Elliptic and Parabolic Conjugacy Classes, and Selberg Super Zeta-Functions. Commun.Math.Phys. 151, 1-37 (1993)

[35] Grosche, C.: Selberg Trace-Formulæ in Mathematical Physics; SISSA-Preprint, SISSA/177/92/FM, October 1992, to appear in: Proceedings of the Workshop "From Classical to Quantum Chaos (18921992)", 21-24 July, 1992, Trieste, World Scientific, Singapore; eds.: G. Dell'Antonio, S. Fantoni and V. R. Manfredi.

[36] Gross, D.J. and Periwal, V.: String Perturbation Theory Diverges. Phys.Rev.Lett. 60, 2105-2108 (1988)

[37] Hejhal, D.A.: The Selberg Trace Formula and the Riemann Zeta Function. Duke Math.J. 43, 441-482 (1976)

[38] Hejhal, D.A.: The Selberg Trace Formula for PSL(2, R), I, Lecture Notes in Mathematics, Vol.548. Berlin, Heidelberg, New York: Springer, 1976

[39] Hejhal, D.A.: The Selberg Trace Formula for PSL(2, R), II, Lecture Notes in Mathematics, Vol.1001. Berlin, Heidelberg, New York: Springer, 1981

[40] Howe, P.S.: Superspace and the Spinning String. Phys.Lett. B 70, 453-456 (1977); Super Weyl Transformations in Two Dimensions. J.Phys.A: Math.Gen. 12, 393-402 (1979)

[41] Jaskolski, Z.: The Polyakov Path Integral over Bordered Surfaces (the Open String Amplitudes). Commun.Math.Phys. 128, 285-318 (1990); The Polyakov Path Integral over Bordered Surfaces (the Closed String Off-Shell Amplitudes). Commun.Math.Phys. 139, 353-376 (1991)

[42] Koyama, S.-Y.: Determinant Expression of Selberg Zeta Functions. I-III. Trans.Amer.Math.Soc. 324, 149-168 (1991); Trans.Amer.Math.Soc. 329, 755-772 (1992); Proc.Amer.Math.Soc. 113, 303$311(1991)$

[43] Losev, A.S.: Calculation of a Scalar Determinant in the Theory of Open Strings. JETP Lett. 48, 330-333 (1988)

[44] Luckock, H.: Quantum Geometry of Strings with Boundaries. Ann.Phys.(N.Y.) 194, 113-147 (1989)

[45] Manin, Yu.I.: The Partition Function of the Polyakov String can be Expressed in Terms of Theta Functions. Phys.Lett. B 172, 184-185 (1986)

[46] Martín-Delgado, M.A. and Mittelbrunn, J.R.: Bordered Riemann Surfaces, Schottky Groups and Off-Shell String Amplitudes. Int.J.Mod.Phys. A 6, 1719-1747 (1991)

[47] Matsumoto, S., Uehara, S. and Yasui, Y.: Hadamard Model on the Super Riemann Surface. Phys. Lett. A 134, 81-86, (1988)

[48] Matsumoto, S., Uehara, S. and Yasui, Y.: A Superparticle on the Super Riemann Surface. J.Math. Phys. 31, 476-501 (1990)

[49] Moore, G., Nelson, P. and Polchinski, J.: Strings and Supermoduli. Phys.Lett. B 169, 47-53 (1986)

[50] Mozorov, A. and Rosly, A.: On Many-Loop Calculations in the Theory of Open Strings. Phys.Lett. B 214, 522-526 (1988); Some Examples of Computation of the Scalar Determinant in Open String Theory. Theor.Math.Phys. 80, 899-911 (1989); and Nucl.Phys. B 326, 185-204 (1989); Strings and Riemann Surfaces; Nucl.Phys. B 326, 205-221 (1989) 
[51] Namazie, M.A. and Rajjev, S.: On Multiloop Computations in Polyakov's String Theory. Nucl.Phys. B 277, 332-348 (1986)

[52] Ninnemann, H.: Holomorphe and Harmonische Formen auf Super-Riemannschen Flächen und ihre Anwendung auf den Fermionischen String. Diploma Thesis, Hamburg University, 1989; Deformations of Super Riemann Surfaces. Commun.Math.Phys. 150, 267-288 (1992)

[53] Ohndorf, T.: Covariant Operator Formalism of the Bosonic String and Polyakov's Path Integral on Bordered Riemann Surfaces. Heidelberg Preprint, HD-THEP-1988-37, 1-140, October 1988

[54] Oshima,K.: Completeness Relations for Maass Laplacians and Heat Kernels on the Super Poincaré Upper Half-Plane. J.Math.Phys. 31, 3060-3063 (1990)

[55] Phillips, R.S. and Sarnak, P.: On Cusp Forms for Cofinite Subgroups of PSL(2, $\mathbb{R})$. Invent.Math. 80, 339-364 (1985)

[56] Polyakov, A.M.: Quantum Geometry of Bosonic Strings. Phys.Lett. B 103, 207-210 (1981)

[57] Polyakov, A.M.: Quantum Geometry of Fermionic Strings. Phys.Lett. B 103, 211-213 (1981)

[58] Rabin, J.M. and Crane, L.: Global Properties of Supermanifolds. Commun.Math. Phys] 100, 141160 (1985); How Different are the Supermanifolds of Rogers and DeWitt?. Commun.Math.Phys. 102, 123-137 (1985); Super Riemann Surfaces: Uniformization and Teichmüller Theory. Commun.Math. Phys. 113, 601-623 (1988)

[59] Rodriguez, J.P.: Open Strings from Closed Strings: Period Matrix, Measure and Ghost Determinant. Phys.Lett. B 202, 227-232 (1988)

[60] Rodriguez, J.P. and Van Tander, A.: Spin Structures for Riemann Surfaces with Boundaries and Cross-Caps. Phys.Lett. B 217, 85-90 (1989)

[61] Rogers, A.: A Global Theory of Supermanifolds. J.Math.Phys. 21, 1352-1364 (1980); On the Existence of Global Integral Forms on Supermanifolds. J.Math.Phys. 26, 2749-2753 (1985); Graded Manifolds, Supermanifolds and Infinite-Dimensional Grassmann Algebras. Commun.Math.Phys. 105, 375-384 (1986)

[62] Schwarz, J.H.: Superstring Theory. Phys.Rep. 89, 223-322 (1982)

[63] Selberg, A.: Harmonic Analysis and Discontinuous Groups in Weakly Symmetric Riemannian Spaces With Application to Dirichlet Series. J.Indian Math.Soc. 20, 47-87 (1956)

[64] Shohkranian, S.: The Selberg-Arthur Trace Formula, Lecture Notes in Mathematics, Vol.1503. Berlin, Heidelberg, New York: Springer, 1992

[65] Sibner, R.J.: Symmetric Fuchsian Groups. Am.J.Math. 90, 1237-1259 (1968)

[66] Steiner, F.: On Selberg's Zeta Function for Compact Riemann Surfaces. Phys.Lett. B 188, 447-454 (1987); Quantum Chaos and Geometry. In: Mitter, H. and Pittner, L.(eds.): Recent Developments in Mathematical Physics, 26.Internationale Universitätswochen, Schladming 1987, 305-312. Berlin, Heidelberg, New York: Springer, 1987

[67] Takhtajan, L.A. and Zograf, P.G.: A Local Index Theorem for Families of $\bar{\partial}$-Operators on Punctured Riemann Surfaces and a New Kähler Metric on Their Moduli Spaces. Commun.Math.Phys] 137, 399-426 (1991)

[68] Uehara, S. and Yasui, Y.: A Superparticle on the "Super" Poincaré Upper Half Plane. Phys.Lett. B 202, 530-534 (1988); Super-Selberg's Trace Formula from the Chaotic Model. J.Math.Phys 29, 2486-2490 (1988)

[69] Venkov, A.B.: On an Asymptotic Formula Connected with the Number of Eigenvalues Corresponding to Odd Eigenfunctions of the Laplace-Beltrami Operator on a Fundamental Region of the Modular Group PSL $(2, \mathbb{Z})$. Sov.Math.Dokl. 18, 524-526 (1977); Selberg's Trace Formula and Non-Euclidean Vibrations of an Infinite Membrane. Sov.Math.Dokl. 19, 708-712 (1979); Selberg's Trace Formula for the Hecke Operator Generated by an Involution, and the eigenvalues of the Laplace-Beltrami Operator on the Fundamental Domain of the Modular Group PSL $(2, \mathbb{Z})$. Math.USSR.Izv. 12, 448$462(1978)$ 
[70] Venkov, A.B.: Spectral Theory of Automorphic Functions, the Selberg Trace Formula, and Some Problems of Analytic Number Theory and Mathematical Physics. Russian Math.Surveys 34, 79-153 (1979)

[71] Venkov, A.B.: Spectral Theory of Automorphic Functions. Proc.Math.Inst.Steklov 153, 1-163 (1981)

[72] Venkov, A.B.: Spectral Theory of Automorphic Functions and Its Applications. Dordrecht: Kluwer Academic Publishers, 1990

[73] Voros, A.: The Hadamard Factorization of the Selberg Zeta Function on a Compact Riemann Surface. Phys.Lett. B 180, 245-246 (1986);

Spectral Functions, Special Functions and the Selberg Zeta Function. Commun.Math.Phys. 110, 439-465 (1987)

[74] Wu, S.: Determinants of Dirac Operators and Thirring Model Partition Functions on Riemann Surfaces with Boundaries. Commun.Math.Phys. 124, 133-152 (1989) 